\title{
Essential role of HCMV deubiquitinase in promoting oncogenesis by targeting anti-viral innate immune signaling pathways
}

\author{
Puja Kumari ${ }^{1}$, Irene Saha ${ }^{2}$, Athira Narayanan ${ }^{1}$, Sathish Narayanan ${ }^{3, *}$, Akinori Takaoka ${ }^{4}$, Nachimuthu Senthil Kumar ${ }^{5}$,
} Prafullakumar Tailor ${ }^{2}$ and Himanshu Kumar, ${ }^{*, 1,6}$

\begin{abstract}
Cancer is a multifactorial disease and virus-mediated carcinogenesis is one of the crucial factors, which is poorly understood. Human cytomegalovirus (HCMV) is a herpesvirus and its components have been evidenced to be associated with cancer of different tissue origin. However, its role in cancer remains unknown. Here, we identified a conserved herpesviral tegument protein known as pUL48 of HCMV, encoding deubiquitinase enzyme, as having a key role in carcinogenesis. We show using deubiquitinase sufficient- and deficient-HCMV that HCMV deubiquitinase is a key in inducing enhanced cellular metabolic activity through upregulation of several anti-apoptotic genes and downregulation of several pro-apoptotic genes expression. Furthermore, HCMV deubiquitinase acquires pro-tumor functions by inhibiting PRR-mediated type I interferon via deubiquitination of TRAF6, TRAF3, IRAK1, IRF7 and STING. Taken together, our results suggest that HCMV infection may promote oncogenesis by inhibiting innate immunity of the host.
\end{abstract}

Cell Death and Disease (2017) 8, e3078; doi:10.1038/cddis.2017.461; published online 5 October 2017

Cancer is a multifactorial disease causing death worldwide and proves to be a burden on human health. DNA viruses such as Epstein-Barr Virus, Hepatitis B Virus, Human Papilloma Virus and Kaposi's Sarcoma-Associated Herpesvirus cause a wide range of malignancies such as nasopharyngeal carcinoma, Burkitt's lymphoma, head and neck cancer, and cervical cancer in the host. ${ }^{1,2}$ In addition, RNA viruses such as Hepatitis C Virus, Human Mammary Tumor Virus, Torque Teno Virus and Human Endogenous Retrovirus also acquire different strategies for oncogenesis. ${ }^{1}$ A growing list of cancerassociated viruses indicates that viral infection can directly or indirectly bring upon carcinogenic state. Human cytomegalovirus (HCMV) is among those DNA viruses that have been found associated with cancers. ${ }^{3,4}$ However, HCMV has not been characterized as an oncogenic virus. HCMV structural components have been found in tumor tissues ${ }^{3-5}$ and reported to be involved in promoting a favorable microenvironment for oncogenic transformation of infected cells. ${ }^{6,7}$ HCMV infects $90 \%$ of the world's population, hence, studying the carcinogenic potential of HCMV and understanding its molecular mechanism may help stop progression of various cancers. Although, HCMV infection is mostly asymptomatic due to host anti-viral immunity, it may lead to oncogenic transformation of normal cells and cancer, when host is immunocompromised owing to immunosuppressive drugs or infection with HIV.
Numerous studies have shown that type I interferons (I-IFNs) have a key role in inhibition of cancer. ${ }^{8-11}$ Virusinfected cells undergo apoptosis as a defense against spread of infection. However, HCMV acquires several strategies to inhibit apoptotic pathway and establish a successful infection. ${ }^{3}$ These survival strategies acquired by HCMV can lead to uncontrolled cell growth.

Here, we identified a novel role of HCMV deubiqutinase (DUB) in oncogenesis. HCMV-DUB is encoded by unique long48 (UL48) gene, a conserved high-molecular-weight protein across the herpesviruses. We have found that HCMV-DUB inhibits expression of various pro-apoptotic genes and induces expression of anti-apoptotic genes. HCMV-DUB enables cells to surpass the G1-phase rapidly and enter into other phases of cell cycle required for cell division. Cellular DUBs have an important role in many signaling pathways, including immune signaling, apoptosis, oncogenesis and developmental pathways. ${ }^{12-14}$ Likewise, our findings as well reveal that upon infection, HCMV-DUB inhibits synthesis of I-IFNs, an anti-cancer factor, by deubiquitinating several signaling molecules such as TNF receptor-associated factor (TRAF)-6 and -3 , interleukin-1 receptor-associated kinase-1 (IRAK1), interferon regulatory factor (IRF)-7 or stimulator of interferon genes (STING) that have a key role in anti-viral innate immunity. Inhibition of I-IFNs by HCMV-DUB correlates with decreased expression of several pro-apoptotic

\footnotetext{
${ }^{1}$ Department of Biological Sciences, Laboratory of Immunology and Infectious Disease Biology, Indian Institute of Science Education and Research (IISER) Bhopal, Bhopal 462066, India; ${ }^{2}$ Laboratory of Innate Immunity, National Institute of Immunology (NII), New Delhi 110067, India; ${ }^{3}$ Department of Biological Sciences, Laboratory of Virology, Indian Institute of Science Education and Research (IISER) Bhopal, Bhopal 462066, India; ${ }^{4}$ Division of Signaling in Cancer and Immunology, Institute for Genetic Medicine, Hokkaido University, Sapporo, Japan; ${ }^{5}$ Department of Biotechnology, Mizoram University, Aizawl 796004, India and ${ }^{6}$ Laboratory of Host Defense, WPI Immunology, Frontier Research Centre, Osaka University, Osaka 5650871, Japan

${ }^{*}$ Corresponding author: H Kumar, Department of Biological Sciences, Laboratory of Immunology and Infectious Disease Biology, Indian Institute of Science Education and Research (IISER) Bhopal, AB-3, Room No. 220, Bhopal By-pass Road, Bhauri, Bhopal 462066, MP, India. Tel: +91 7556691413 ; Fax: +91 7556692392 ; E-mail: hkumar@iiserb.ac.in

Deceased.

Received 21.2.17; revised 06.7.17; accepted 24.7.17; Edited by M Herold
} 
a

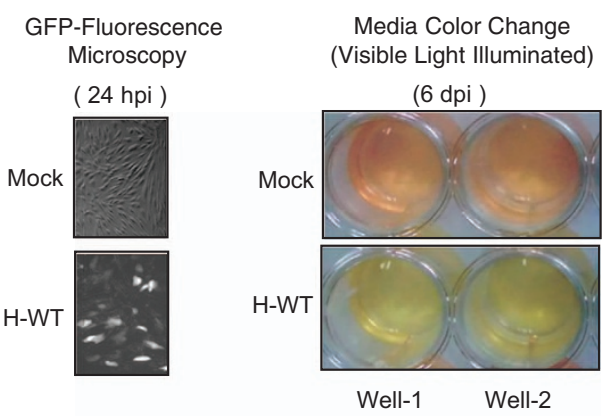

$\mathbf{f}$

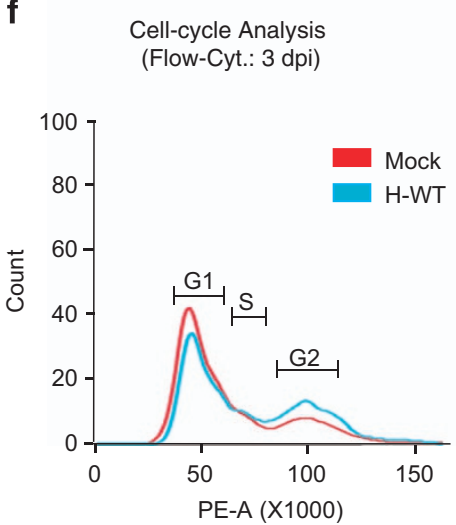

C

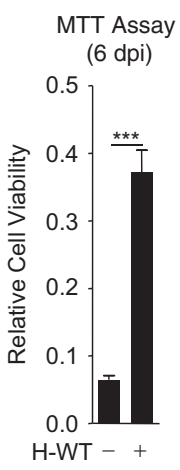

d

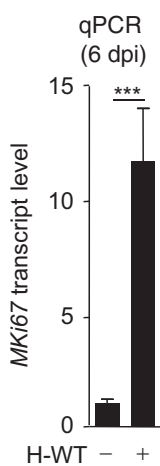

e

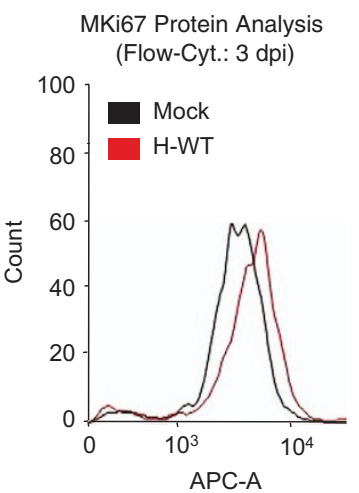

MKi67 Protein Analysis (Flow-Cyt.: 3 dpi), summary

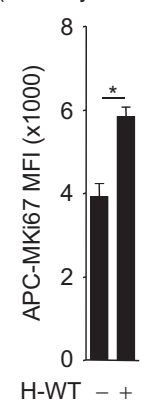

$g$

Cell-cycle Analysis (Flow-Cyt.: 3dpi, summary)

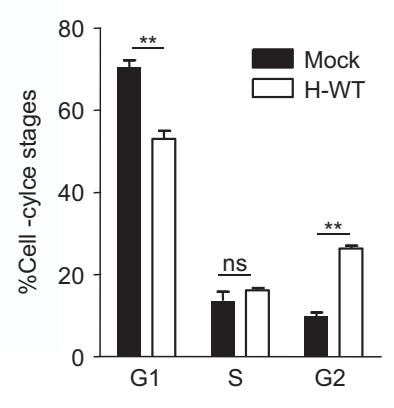
\begin{tabular}{lll}
\multicolumn{3}{c}{ Anti-apoptotic genes } \\
\hline qPCR & qPCR & qPCR \\
(6 dpi) & (6 dpi) & (6 dpi)
\end{tabular}

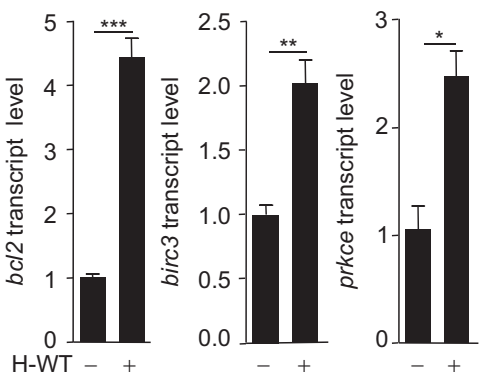

\begin{tabular}{|c|c|c|c|}
\hline HFFs & G1 (\%) & S (\%) & G2 (\%) \\
\hline Mock & 65.7 & 10.4 & 15.8 \\
\hline H-WT & 51 & 16.8 & 27 \\
\hline
\end{tabular}

Figure $1 \mathrm{HCMV}$ infection induces oncogenic properties in non-transformed HFFs. (a) GFP expression was observed under the fluorescence microscope at $24 \mathrm{~h}$ post infection (hpi) with GFP-tagged WT-HCMV (H-WT) in HFFs. (b) Culture media color change (red to yellow) was visually observed on day 6 post infection (dpi), in HFFs infected with H-WT. (c) Cell viability (metabolic activity) of mock or H-WT-infected HFFs was quantified by MTT assay, on 6 dpi. (d) Transcript and (e) protein of MKi67 was quantified by qPCR and flow cytometer respectively, in mock, H-WT-infected HFFs, (f, left, right, bottom) flowcytometry was done for mock and H-WT-infected HFFs by staining them with propidium iodide ( $\mathrm{PI}$, shown in $x$ axis) on 3 dpi, to detect cell cycle stages upon HCMV infection and (g) Anti-apoptotic gene (bcl2, birc3 and prkce) status was compared by qPCR in mock or H-WT infected HFFs on 6 dpi. Shown results are the representative of three $(\mathbf{a}-\mathbf{d}, \mathbf{g})$ or two $(\mathbf{e}, \mathbf{f})$ independent experiments. (f) Statistical analysis was done with data of two independent experiments. Differences were considered statistically significant with a ${ }^{*} P$-value $<0.05,{ }^{* *} P$-value $<0.01$ and ${ }^{* \star *} P$-value $<0.001$, ns, non-significant difference $(P$-value $>0.05)$

genes and increased expression of anti-apoptotic genes, which also indicates its oncogenic potential during infection.

\section{Results}

HCMV induces oncogenic properties. Association of HCMV antigens with various cancer types is well known. However, whether HCMV promotes cancer upon infection is unknown. To investigate the role of HCMV in oncogenesis, non-transformed human foreskin fibroblasts (HFFs) were infected with a GFP-tagged laboratory strain of wild-type HCMV (WT-HCMV), AD169 (MOI 5). On second day post infection (dpi), virus infection was observed by GFP fluorescence (Figure 1a). On sixth dpi, infected cells showed a characteristic cytopathic effect (CPE) and also change in growth media color (red to yellow) (Figure 1b). The color change owing at least in part to release of marker GFP from infected cells but also may indicate enhanced metabolic activity during infection. To evaluate the possibility more directly, we performed an MTT assay comparing infected cells with uninfected. A fourfold higher metabolic activity was observed for WT-HCMV-infected cells compared with uninfected cells (Figure 1c). In addition, an enhanced level of RNA and protein of MKi67, a cell proliferation marker gene, was observed in WT-HCMV-infected HFFs compared with uninfected HFFs (Figures 1d and e), consistent with the result of MTT assay. This led us to further investigate the cell cycle stages of HCMV-infected cells by flowcytometry, which showed a detectable skewing pattern toward G2-phase in infected cells than in uninfected cells (Figure 1f). 
The majority of viral infection leads to cell death, as a protective innate immune response to reduce viral-load within the host. The cell death is triggered through activation of apoptotic pathways, however, HCMV infection increased cell survival via significantly enhancing the expression of antiapoptotic genes such as bcl2, birc3 and prkce, compared with mock infection (Figure 1g), indicating that HCMV infection protects cells from apoptosis and promotes cell survival. During cancer progression, expression of anti- and proapoptotic genes is modulated to support cancer progression. Most often increase in expression of anti-apoptotic genes and decrease in expression of pro-apoptotic genes favor cancer progression. Collectively, these results indicate that HCMV infection induces oncogenic properties in non-transformed cells through upregulation of anti-apoptotic genes, resulting into enhanced cell proliferation.

DUB activity of HCMV-pUL48 induces oncogenic properties. Previously, it has been reported that the inactive DUB of Marek's disease herpesvirus compromises the ability of that virus to cause lymphoma in chickens. ${ }^{15}$ In addition, reports suggest that deregulation of cellular DUBs could also promote various cancer types in humans and mouse. ${ }^{16-18}$ However, HCMV-DUB and its role in oncogenesis are not known. We hypothesized that HCMV-DUB may also have such a role. To test this, we used a previously reported HCMV mutant virus that encodes an inactive DUB. ${ }^{19}$ After infecting HFFs with equal amount of WT and DUB-mutant ( $\triangle D U B$ ) GFP-tagged HCMV, an equal amount of virus infection (GFP Fluorescence) was observed on second dpi (Figure 2a). On sixth dpi, $\triangle$ DUB-HCMV-infected cells showed much lessmetabolic activity than WT-HCMV-infected cells, however, comparable to mock infected cells (Figure 2b). To confirm the differential responses of WT- and $\triangle \mathrm{DUB}-\mathrm{HCMV}$, MTT assay and MKi67 transcript and protein analysis were performed for cell metabolic activity and cell proliferation, respectively, which indicated that $\triangle \mathrm{DUB}-\mathrm{HCMV}$ reduced cell metabolic activity and proliferation compared with WT-HCMV (Figures 2c-e). Furthermore, HFF cell counting and cell cycle analysis after three dpi with HCMV, revealed that WTHCMV infection causes enhanced cell proliferation (Figure 2f) and more cell accumulation in the G2-phase compared with mock or $\triangle \mathrm{DUB}-\mathrm{HCMV}$ infection (Figure $2 \mathrm{~g}$ ).

Next, to confirm that HCMV-DUB can stimulate cell proliferation, we created as previously reported a mammalian expression plasmid, ${ }^{20}$ encoding amino acid 1-1162 of pUL48, a functional DUB domain (UL48N) and UL48N lacking DUB function (UL48NADUB) of HCMV. The IMR32 cells (a noninvasive neuroblastoma cell line) stably expressing UL48N, UL48N $\triangle$ DUB or empty vector $(\mathrm{Vec})$ were generated and subjected to cell proliferation analysis from Day 0 to Day 6 after seeding at a density of $0.1 \times 10^{6}$ cells and by counting the number of cells on every alternate day. The IMR32 cells, stably expressing WT-UL48N showed rapid proliferation than those stably expressing either $\triangle \mathrm{DUB}-U L 48 \mathrm{~N}$ or Vec (Figure $2 \mathrm{~h}$ ). This was further confirmed by cell cycle analysis of IMR32 cells, which showed an enhanced accumulation of UL48N expressing IMR32 cells in S- and G2-phases compared with Vec and UL48N $\triangle$ DUB (Figure 2i). Collectively, these results indicate that HCMV-DUB can induce cell proliferation and UL48-DUB is crucial for this activity.

HCMV-DUB induces cancer hallmarks. The majority of viral infections lead to cell death through apoptotic pathways, which is a protective innate immune response. To understand whether HCMV-DUB modulates pro-apoptotic and antiapoptotic gene expressions during infection, WT-HCMV and $\triangle$ DUB-HCMV-infected HFFs were first analyzed for the expression of anti- and pro-apoptotic genes through qPCR, on 6th dpi. Transcription of anti-apoptotic genes such as bcl2, birc3, prkce, survivin and xiap was significantly increased by many folds, in WT-HCMV-infected HFFs, however the transcript level remained either unchanged or reduced in $\triangle$ DUB-HCMV-infected HFFs compared with mock (Figure 3a). Furthermore, protein-expression analysis of anti-apoptotic genes-bcl2, birc3 and survivin-showed an increased expression in WT-HCMV-infected HFFs than in mock or $\triangle \mathrm{DUB}-\mathrm{HCMV}$-infected HFFs (Figure 3c). Expression level of few other anti-apoptotic genes such as ciap1, cflip and $\mathrm{mcl}-1$ decreased in case of both, WT and $\triangle \mathrm{DUB}-\mathrm{HCMV}$ infection compared with mock infection (Supplementary Figures S1a-S1c), which suggests HCMV-DUB has no specific role in regulating these anti-apoptotic genes. Surprisingly, transcript level of $b c l-x l$ anti-apoptotic gene was reduced owing to WT-HCMV infection, whereas remained unchanged upon $\triangle \mathrm{DUB}-\mathrm{HCMV}$ infection (Supplementary Figure S1d). Similarly, transcription of proapoptotic genes such as trail, $R b, p 53$, fadd and tnfa, remained either unchanged, less induced or reduced in WTHCMV-infected cells compared with mock, whereas expression of these pro-apoptotic genes was higher in the $\triangle D U B-$ HCMV-infected cells (Figure 3b). The p53 protein was induced upon infection with both WT- and $\triangle \mathrm{DUB}-\mathrm{HCMV}$, however induction was comparatively less in WT-HCMVinfected HFFs (Figure 3c). Transcription of few other proapoptotic genes such as bad and bax was decreased upon infection with both, WT- and $\triangle \mathrm{DUB}-\mathrm{HCMV}$, suggesting no specific role of HCMV-DUB in regulating bad and bax, whereas transcription of caspase-8, and p21 was reduced only in WT-HCMV-infected HFFs, and remained unchanged in $\triangle$ DUB-HCMV-infected HFFs (Supplementary Figures S1fS1i). Furthermore, protein analysis of p21 and caspase-3 showed a decreased expression in WT-HCMV-infected cells compared with mock (Figure 3c). The expression of several other anti- and pro-apoptotic genes was tested, with no evidence of differences between WT- and $\triangle \mathrm{DUB}-\mathrm{HCMV}$ infected cells compared with mock (Supplementary Figures S1e, S1j-S1I) suggesting no involvement of HCMV infection to regulation of these genes. Differential expression of antiand pro-apoptotic genes during WT-HCMV- and $\triangle \mathrm{DUB}$ HCMV infection indicates that HCMV-pUL48 DUB activity interferes with the induction of apoptosis and may thus induce oncogenic properties. Consistent with this interpretation, we found that the ability of anti-cancer drug etoposide to promote apoptosis, was reduced by WT-HCMV infection but not by $\triangle$ DUB-HCMV infection (Figure 3d).

Next, we tested uptake of glucose, as cancer cells require increased glucose for metabolism and cell division. ${ }^{21}$ To this end, IMR32 cells stably expressing Vec, UL48N and 
4

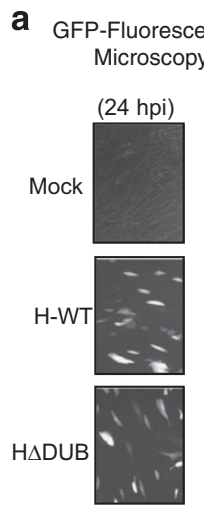

MKi67 Protein Analysis
(Flow-Cyt.: 3 dpi)
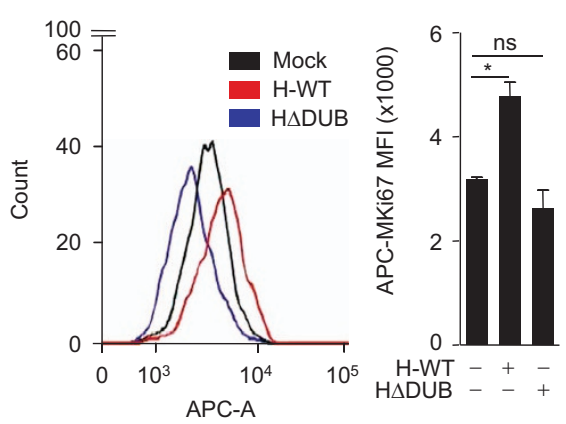

$\%$ of HCMV infection Flow-Cyt.: 24 hpi MFI Summary

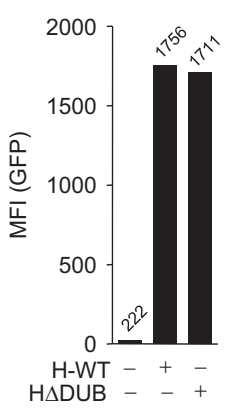

f HFF cell-counting (3 dpi)

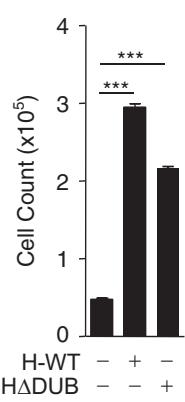

b

Media Color Change
(Visible Light Illuminated)

C

MTT Assay

(6 dpi)
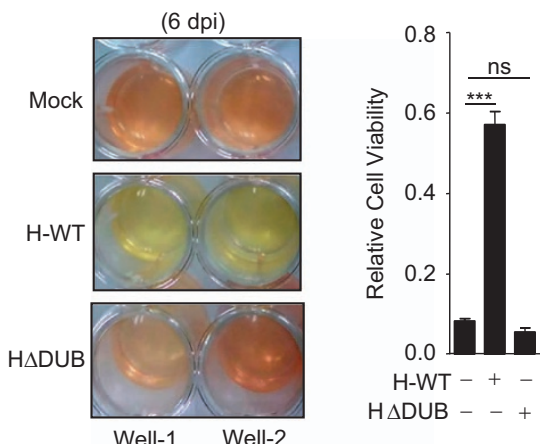

$\mathrm{H} \triangle \mathrm{DUB}-{ }_{-}+$ d

qPCR

(6 dpi)

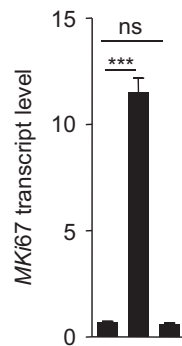

H-WT -+ H $\triangle$ DUB - - + g

HFF Cell-cycle Analysis (Flow-Cyt.: 3 dpi)

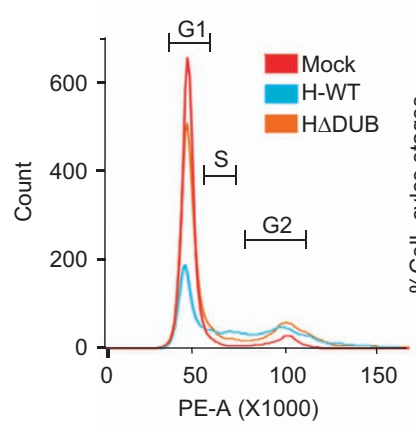
(Flow-Cyt.: 3dpi, summary)

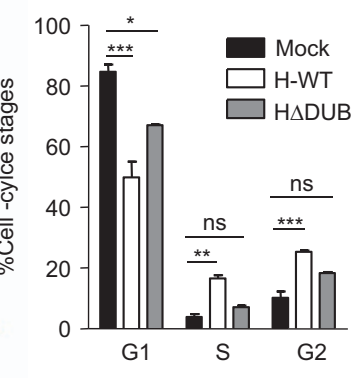

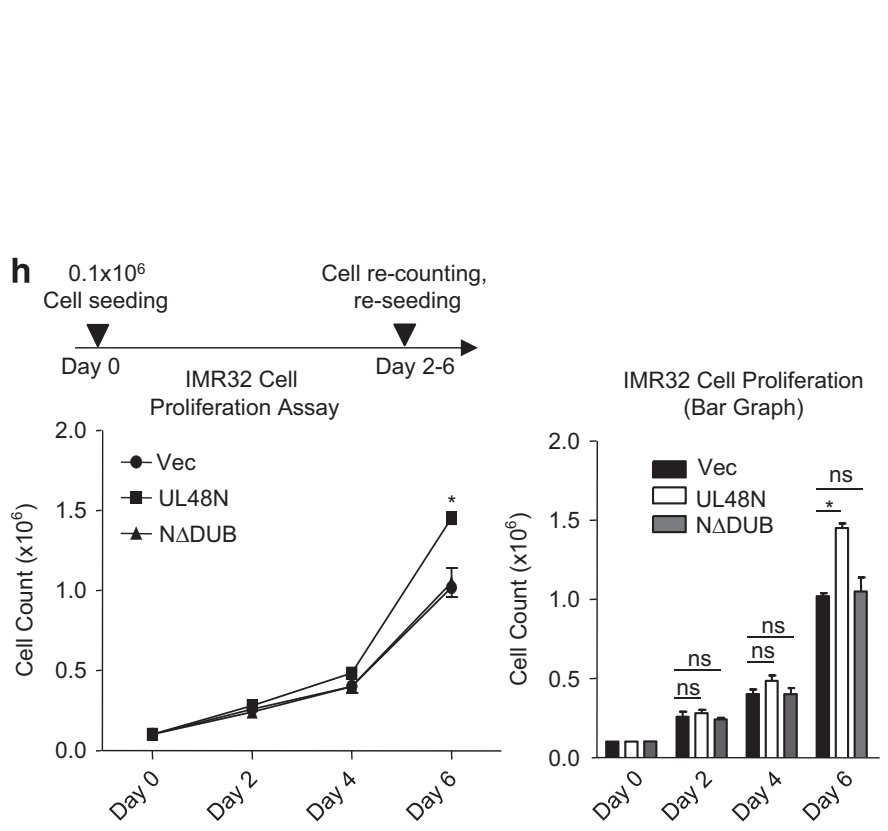

\begin{tabular}{|c|c|c|c|}
\hline HFFs & G1 (\%) & $\mathbf{S ~ ( \% ) ~}$ & G2 (\%) \\
\hline Mock & 87 & 2.99 & 8.14 \\
\hline H-WT & 44.8 & 17.6 & 24.9 \\
\hline H $\triangle$ DUB & 67.3 & 6.71 & 18 \\
\hline
\end{tabular}

i

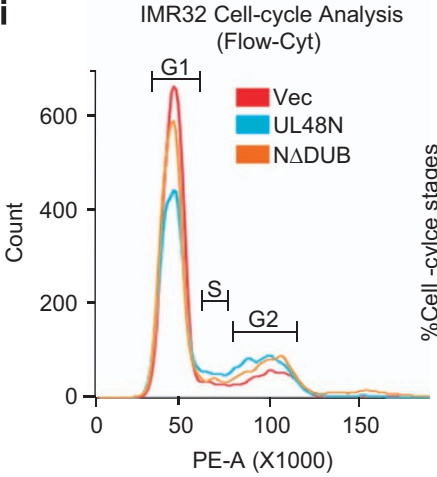

IMR32 Cell-cycle Analysis (Flow-Cyt.: summary)
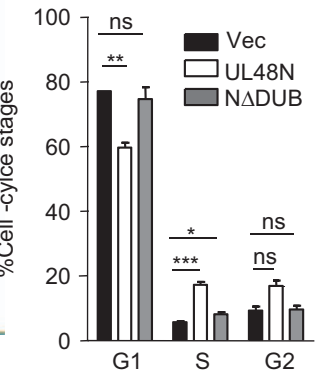

\begin{tabular}{|c|c|c|c|}
\hline HFFs & G1 (\%) & $\mathbf{S}(\%)$ & G2 (\%) \\
\hline Vec & 77.1 & 5.98 & 10.5 \\
\hline UL48N & 58.2 & 16.5 & 18.6 \\
\hline N $\triangle$ DUB & 71 & 7.5 & 10.8 \\
\hline
\end{tabular}

UL48N $\triangle$ DUB were glucose starved and treated with FITClabeled glucose (2-NBDG). Flow cytometric analysis revealed that cells expressing UL48N took-up more 2-NBDG than those expressing Vec or UL48NADUB (Figure 3e). Moreover, overexpression of UL48N, increased the cell migration (Figure 3f) and tissue invasion (Figure 3g) of IMR32 cells (tested by wound-healing and matrigel invasion assay, respectively), whereas UL48NADUB did not induce any of 
Figure 2 HCMV deubiquitinase is responsible for induction of oncogenic properties. (a) HFFs were infected with equal MOI of GFP-tagged H-WTand H $\triangle D$ DUB; equal amount of GFP expression was microscopically observed at $24 \mathrm{~h}$ post infection (hpi) with GFP-tagged H-WT and H $\Delta$ DUB in HFFs. Quantification of GFP fluorescence (HCMV infection) was done with flow cytometer; histogram shows the percentage of HCMV infection at 24 hpi, measured by flowcytometry and bar- graph shows the GFP-Mean Fluorescent Intensity (MFI) of the respective histograms. (b) Culture media color change (red to yellow) was visually observed on 6 dpi, in HFFs infected with H-WT. (c) Cell viability (metabolic activity) was quantified and compared among mock, H-WT and H $\triangle$ DUB-infected HFFs, by MTT assay. (d) Transcript of proliferation marker gene MKi67 was quantified by qPCR in mock, H-WT and H $\triangle$ DUB-infected HFFs. (e, left) MKi67 protein analysis was done in mock, H-WT and HDUB-infected HFFs by flowcytometry (e, right) MFI was calculated for respective MKi67 histograms. (f) Proliferation rate of Mock, H-WTand H $\Delta$ DUB-HCMV-infected HFFs was analyzed by seeding them into equal number, harvesting them on 3 dpi and counting them to compare the total number of cells. (g, left, right, bottom) Cell Cycle stages of mock, H-WT and H $\Delta$ DUB-infected HFFs were analyzed by flowcytometry by staining these HFFs with propidium iodide (PI) (shown in $x$ axis) on 3 dpi. (h, left, right) The cell proliferation rate of IMR32 cells, stably expressing Vec, UL48N and UL48N $\Delta$ DUB, was compared by initially seeding them into a density of $0.1 \times 10^{6}$ cells, followed by counting and re-seeding them on every alternate day for 6 days. (i, left, right) Cell Cycle stages IMR32 stably expressing vector UL48N or N $\triangle$ DUB were analyzed by flowcytometry by staining the cells with PI. dpi: days post infection, Vec: Empty Vector, N $\Delta$ DUB: UL48N $\Delta$ DUB. Shown results are the representative of three $(\mathbf{a}-\mathbf{d}, \mathbf{f}, \mathbf{h}, \mathbf{i})$ or two $(\mathbf{e}, \mathbf{g})$ independent experiments. $(\mathbf{g}, \mathbf{h})$ Statistical analysis was done with data of two $(\mathbf{g})$ and three (h) independent experiments and the difference was calculated between mock versus WT-HCMV infection or mock versus H $\Delta$ DUB-HCMV infection (g) or between Vec versus UL48N or Vec versus N $\triangle$ DUB (h). Differences were considered statistically significant with a ${ }^{*} P$-value $<0.05$, ${ }^{* *} P$-value $<0.01$ and ${ }^{* \star} P$-value $<0.001$, ns, non-significant difference $(P$-value $>0.05)$

these characteristics of cancer. These data suggest that HCMV-DUB could promote several oncogenic properties and possibly initiate or promote cancer.

\section{HCMV-DUB inhibits anti-viral innate immunity for onco-} genesis. Viral infections can induce cellular anti-viral innate immune responses, including I-IFNs, responsive to pattern recognition receptors (PRRs). Besides anti-viral immunity, the I-IFNs are also known to have a key role in elimination of selfaltered or tumor cells through induction of pro-apoptotic genes such as $p 53^{22} R b^{23,24}$ trail $^{10,25}$ and suppression of anti-apoptotic genes such as prkce, ${ }^{10,26}$ birc $3^{10}$ and $b c / 2 .^{10,27}$ When we tested for expression of I-IFNs (pan-IFNa and IFN $\beta$ ) in HFFs, infected with either WT or $\triangle D U B-H C M V$, or transfected with poly(l:C), as a known inducer only, $\triangle \mathrm{DUB}$ HCMV and poly(l:C), significantly increased the transcript levels of pan-IFNa (Figure 4a) and IFN $\beta$ (Figure 4b) at all tested time points, compared with WT-HCMV. Interestingly, the expression-pattern of many of the pro-apoptotic and antiapoptotic genes aligned with the expression-pattern of I-IFNs by WT- and $\triangle$ DUB-HCMV (e.g., compare transcription patterns in Figures $3 a$ and $b$ and Figures $4 a$ and $b$ ), which suggests that inhibition of pro-apoptotic and induction of antiapoptotic gene expression by WT-HCMV infection, could be due to inhibition of I-IFN synthesis.

HCMV induces innate immunity through several PRRs, including endosome localized TLR9 and cytosolic DNA sensors (CDSs). ${ }^{28-32}$ TLR9 and CDSs, relay their signals through Myeloid differentiation primary response gene88 (MyD88) and STING, respectively, for the synthesis of I-IFNs. ${ }^{33-35}$ To understand the role of HCMV in I-IFN synthesis and its dependence on MyD88- or STING-pathway, these genes were knocked down in HFFs using short-hairpin RNA (shRNA), wherein myd88 and sting transcripts were significantly reduced compared with control shRNA for GFP (Supplementary Figures S2a-S2b). HFFs deficient in either myd88 or sting were mocked or infected with WT-HCMV for analysis of I-IFN synthesis. WT-HCMV induced the synthesis of IFN $\beta$ in control HFFs whereas reduced the synthesis in mydd 88 or sting knocked down HFFs (Figures $4 \mathrm{c}$ and d). This observation suggests that in absence of either MyD88 or STING, induction of I-IFN synthesis is compromised during HCMV infection, which further confirms a dependency on MyD88 and STING for I-IFN synthesis, during HCMV infection.
To understand the role of HCMV-DUB (UL48N) in I-IFN synthesis, genes for MyD88 or STING were paired with UL48N and co-expressed in HEK293 cells together with the luciferase reporter gene regulated either by the IFNa4, IFNa6 or IFN $\beta$ promoter. All I-IFN promoters increased luciferase production when co-expressed with either the MyD88 or STING gene, however, in all cases the increase was inhibited upon co-expression of UL48N. (Figures $4 \mathrm{e}-\mathrm{g}$ and Supplementary Figure S2c). UL48N overexpression also inhibited CpG (TLR9-Ligand)-stimulated transcription of I-IFN and IP10 in HeLa cells (Figures $4 \mathrm{~h}$ and $\mathrm{i}$ ). Furthermore, we tested the efficacy of HCMV-DUB in affecting the I-IFN-dependent antiviral immunity by transfecting IMR32 cells with UL48N or UL48N $\triangle$ DUB constructs, followed by infecting the cells with New Castle Disease Virus (NDV), $24 \mathrm{~h}$ post transfection (hpt). Testing for NDV-replication by quantitative (q)-PCR, showed marked increase in NDV-replication in cells transfected with UL48N but not with UL48NADUB (Figure 4j). This finding suggests that UL48N suppressed cellular anti-viral responses, which was further proved by analyzing the replication status of both WT and $\triangle$ DUB-HCMV in HFFs wherein replication of WTHCMV was gradually increased with time compared with $\triangle$ DUB-HCMV (Supplementary Figure S2d).

Further, to investigate the role of HCMV-DUB in antagonizing I-IFN-induced cell death, HCMV-infected HFFs were treated with recombinant-IFN $\beta$ ( $r$ IFN $\beta$ ) on third dpi. An MTT assay, done 3 days after rIFN $\beta$ treatment, showed antagonization of rIFN $\beta$-mediated cell death by WT-HCMV-infected HFFs (Figure 4k). To further analyze I-IFN-antagonizing ability of UL48N for oncogenesis, IMR32 cells were transfected with different amounts of plasmid encoding UL48N or UL48N $\triangle$ DUB and wound was created $24 \mathrm{hpt}$. Cell monolayer with wounds were treated with rIFN $\beta$ and observed after $72 \mathrm{~h}$ for wound healing. The UL48N-transfected IMR32 cells showed dosedependent wound-healing compared with cells transfected with UL48N $\triangle D U B$ or $V e c$, and cells treated with rIFN $\beta$ only (Figure 4I). Amount of FLAG-tagged UL48N and UL48NADUB was measured by immunoblotting (Figure 4l). Finally, to investigate a correlation of I-IFN inhibition with induction of oncogenic properties, receptor for I-IFNs (IFNAR) on HFFs was blocked using anti-IFNAR2 antibody to ultimately inhibit positive feedback signaling. Following blockade of IFNAR, HFFs were infected with WT- or $\triangle \mathrm{DUB}-\mathrm{HCMV}$ and $3 \mathrm{dpi}$, transcript status of IFN $\beta$ was analyzed that showed an 


\section{6}

a

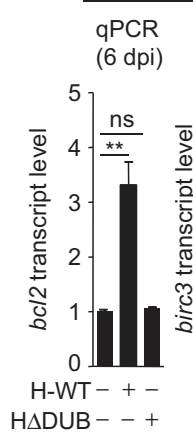

Anti-apoptotic genes

$\begin{array}{llll}\text { qPCR } & \text { qPCR } & \text { qPCR } & \text { qPCR } \\ (6 \mathrm{dpi}) & (6 \mathrm{dpi}) & (6 \mathrm{dpi}) & (6 \mathrm{dpi})\end{array}$

b

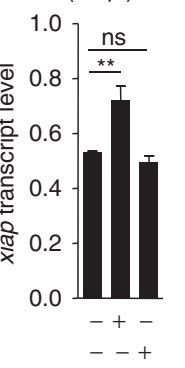

b $\quad$ Pro-apoptotic genes

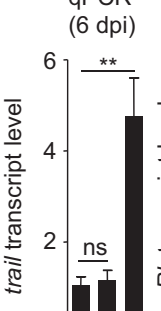

(6 dpi)

qPCR
$(6 \mathrm{dpi})$
$\begin{array}{rr}\text { qPCR } & \text { qPCR } \\ \text { (6 dpi) } & \text { (6dpi) }\end{array}$
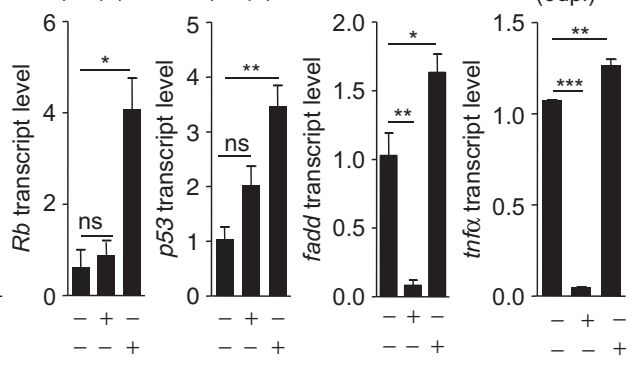

$\mathrm{H}-\mathrm{WT}-+-$

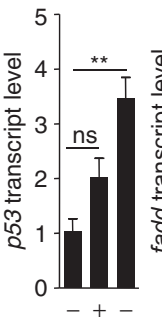

C

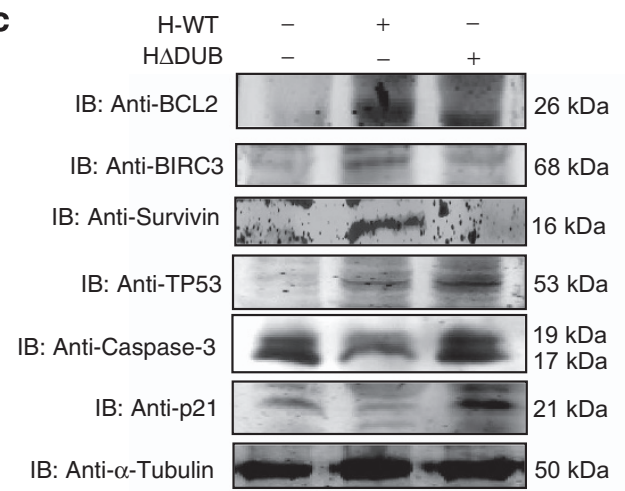

d

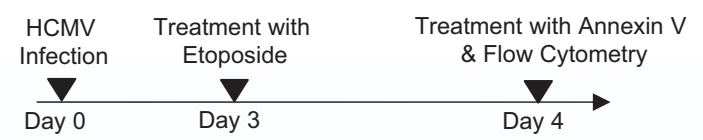
$\mathrm{H} \Delta \mathrm{DUB}--+$

e Seeding of

Addition of serum-

IMR32 stable cells glucose free media \& Flow Cytometry

Treatment with 2-NBDG
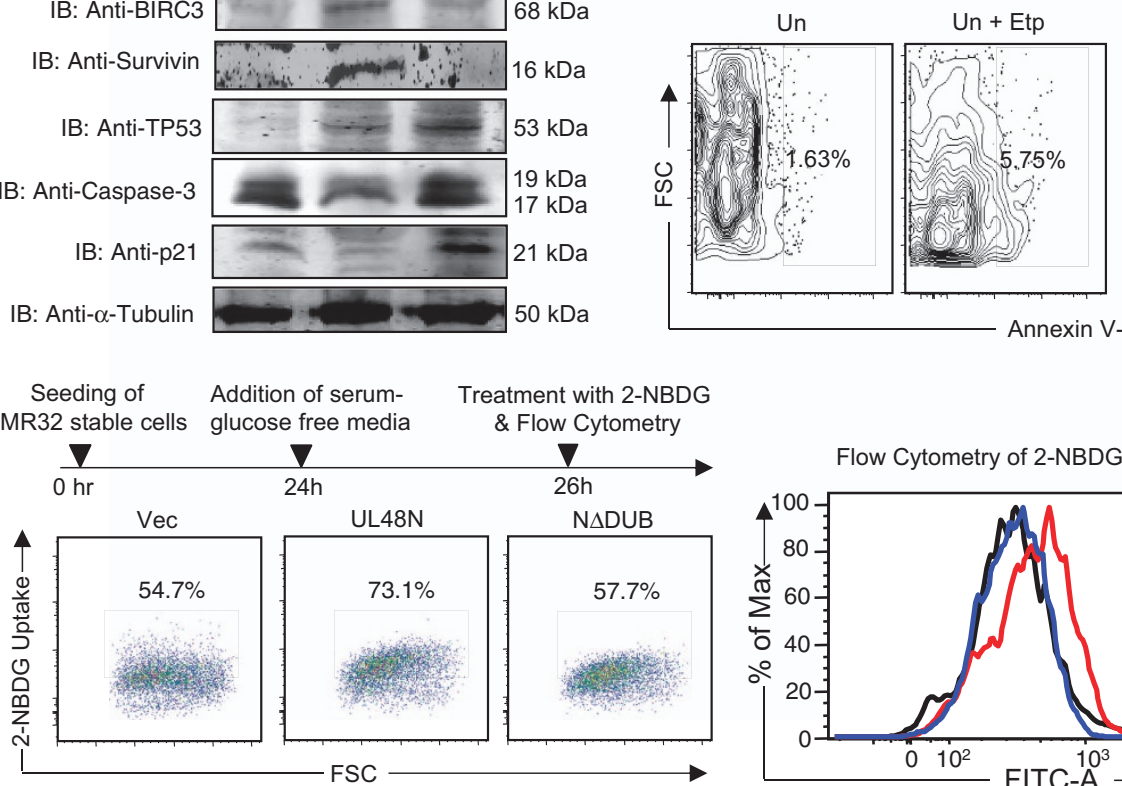

H-WT + Etp

$\mathrm{H} \Delta \mathrm{DUB}+\mathrm{Etp}$

- Annexin V-PE
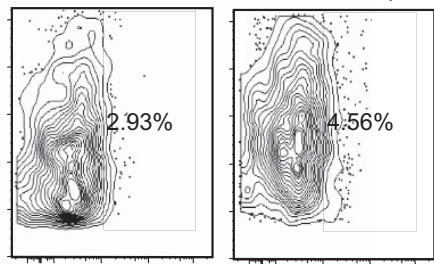

Flow Cytometry of 2-NBDG (FITC-labeled) uptake

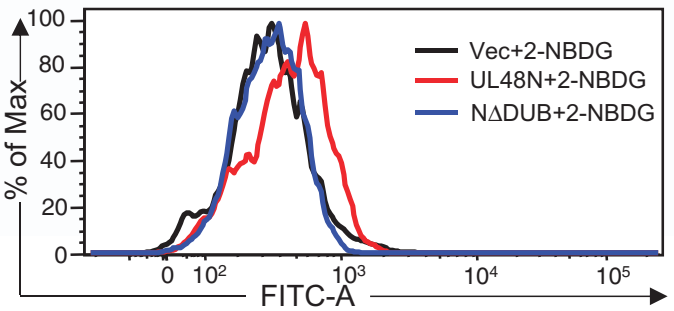

f
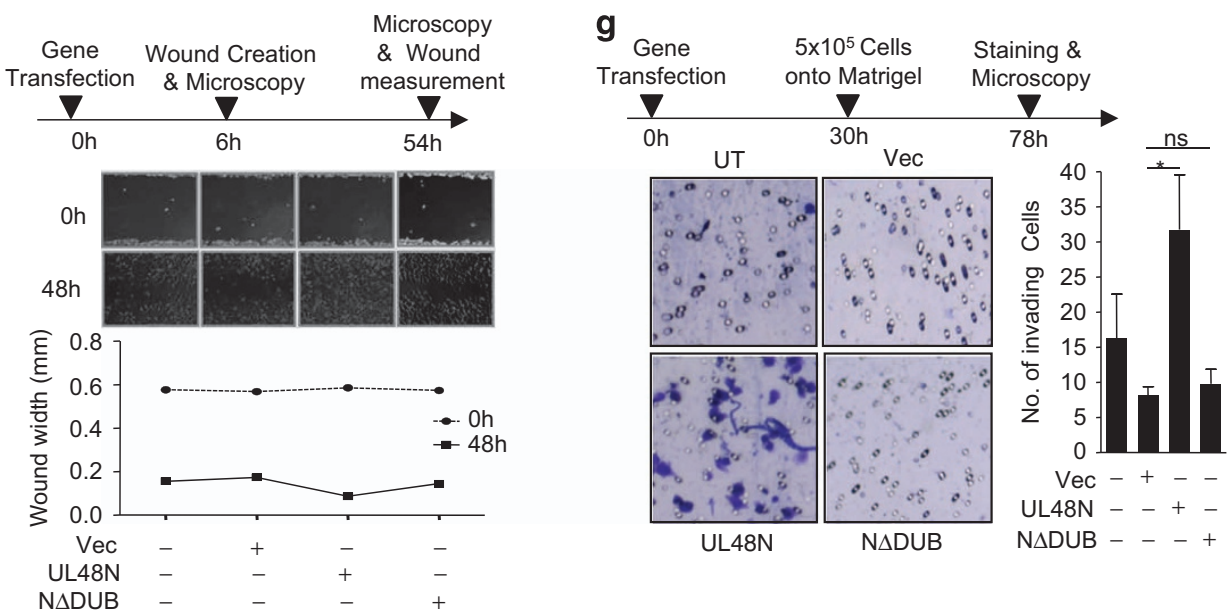

inhibition upon IFNAR blockade (Figure 4m). The cell proliferation and viability status were analyzed with same cells by doing MKi67 transcript quantification and MTT assay, respectively. Cells treated with anti-IFNAR2 antibody showed enhanced proliferation (Figure $4 \mathrm{n}$ ) and viability (Figure 40) compared with antibody-untreated cells that inversely correlated with IFN $\beta$ expression in same cells (Figures 4m-o). This suggests that induction of oncogenic properties upon 
Figure 3 HCMV deubiquitinase induces cancer hallmarks: (a, b) HFFs were infected with equal MOI of GFP-tagged WT-HCMV (H-WT) and $\triangle D U B-H C M V ~(H \triangle D U B)$ and analyzed for apoptosis-associated genes on $6 \mathrm{dpi}$, (a) transcript level of anti-apoptotic genes bcl2, birc3, prkce, survivin and xiap as well as (b) transcript level of pro-apoptotic genes trail, Rb, p53, fadd and tnfo was measured by qPCR in mock, H-WTand H $\triangle$ DUB-infected HFFs. (c) Endogenous protein level of BCL2, BIRC3, Survivin, TP53, Caspase-3 and p21 was analyzed in HFFs upon 8 dpi with H-WT and H $\Delta$ DUB (d) HCMV-infected HFF cells were treated with 30 $\mu$ M etoposide on 3 dpi and analyzed for apoptosis rescue $24 \mathrm{~h}$ later by quantifying Annexin V level through flow cytometer. (e, left, right) IMR32 cells stably expressing Vec, UL48N and UL48N $\Delta$ DUB were glucose starved for $2 \mathrm{~h}$ and treated with FITC-labeled Glucose for $10 \mathrm{~min}$ and then subjected to flow cytometer to observe the glucose uptake (f, top) Wound was created in IMR32 cells transiently transfected with Vec, UL48N and UL48N $\triangle$ DUB expression plasmids as depicted and healing was observed for $48 \mathrm{~h}$, (f, bottom) wound size was measured. (g, left) $5 \times 10^{5}$ IMR32 cells transiently transfected with Vec, UL48N and UL48N $\triangle$ DUB expression plasmids were resuspended in serum deprived growth media and inoculated onto the matrigel layer containing insert, which was further kept in contact with serum containing growth media. Tissue invasion of these IMR32 cells was analyzed through matrigel invasion. (g, right) Matrigel invaded cells were counted and graph was plotted. Blue dots represent the invaded cells. Vec: Empty Vector, dpi: days post infection, N $\Delta$ DUB: UL48N $\Delta D U B$, Etp: Etoposide, FITC-A: FITC-Area. Shown results are the representative of three $(\mathbf{a}, \mathbf{b}, \mathbf{d}, \mathbf{e}, \mathbf{f}, \mathbf{g})$ or single $(\mathbf{c})$ independent experiments. Differences were considered statistically significant with a ${ }^{*} P$-value $<0.05,{ }^{\star \star} P$-value $<0.01$ and ${ }^{\star \star \star} P$-value $<0.001$, ns, non-significant difference $(P$-value $>0.05)$. See also Supplementary Figure $S 1 a-S 11$

WT-HCMV infection is owing to inhibition of I-IFNs. In addition, to understand the reason behind enhanced G2-phase entry of WT-HCMV-infected cells, cyclin-D1 transcript level was measured in HCMV-infected HFFs after 2 dpi, as I-IFN inhibits cyclin-D1 expression to block cell cycle at G1-phase. ${ }^{36-45}$ Interestingly, cyclin-D1 (ccnd1) expression was upregulated upon WT-HCMV infection compared with mock or $\triangle \mathrm{DUB}$ HCMV infection to HFFs (Supplementary Figure S2e), which inversely correlated with the I-IFN results observed in Figures $4 \mathrm{a}$ and $\mathrm{b}$. These data collectively suggest that HCMV-DUB could inhibit I-IFN synthesis and I-IFN-mediated death of infected cells and thus promote oncogenic properties.

HCMV-DUB deubiquitinates cytoplasmic molecules to inhibit I-IFN synthesis and promote carcinogenic properties. The pUL48 contains nuclear localization sequences (NLS), suggesting that differential localization of pUL48N within the cell may be critical for its DUB function and I-IFN inhibition. To test this, NLS-mutant of UL48N was created as described previously ${ }^{46}$ and named as UL48N $\Delta$ NLS (Figure 5a), which was unable to enter the nucleus (Figure 5b) and was equally efficient DUB as UL48N (Figure 5c). To analyze the function of UL48N (cytoplasmic or nuclear function) in I-IFN synthesis pathways, I-IFN promoter activity was measured with overexpression of UL48N and UL48N $\triangle N L S$. To this end, IFNa4 and IFN $\beta$ promoter was activated by co-expression of MyD88 or STING, which are the key molecules of TLR9 or CDS signaling pathways, respectively. UL48N and UL48N $\triangle N L S$, both inhibited MyD88 or STING-mediated IFNa4 or IFN $\beta$ promoter activity, respectively (Figures $5 d$ and e). We hypothesized that UL48N deubiquitinates the cytoplasmic (not the nuclear) signaling molecules activated by PRRs, whose ubiquitination is necessary for induction of I-IFNs. We also compared UL48N $\triangle$ NLS with UL48N in a wound-healing assay and found that wounds of IMR32-monolayers expressing either of the constructs, closed with equal efficiency after $48 \mathrm{~h}$, (Figure 5f), despite a modest difference in I-IFN inhibiting property. This indicates that NLS of pUL48 is dispensable for the HCMV-DUB induced cell migration observed above (e.g., Figure 3f). These results show that nuclear localization of UL48N is not required to show inhibitory effect on I-IFN synthesis and cellular migratory activities.
HCMV-DUB deubiquitinates PRR-mediated signal transducers to inhibit I-IFN synthesis. To facilitate the synthesis of I-IFNs through PRRs, many signaling mediators get K63 (lysine-63) ubiquitinated (Figure 6a). As K63 ubiquitination of cytoplasmic molecules is an important event during innate immune signaling, our next approach was to find out the cytoplasmic target molecule whose deubiquitination is facilitated by UL48N and is necessary for inhibition of I-IFN synthesis. To this end, various signaling molecules of the TLR and CDS pathways, undergoing K63 ubiquitination for induction of I-IFNs synthesis, were co-expressed with UL48N or UL48N $\triangle$ DUB in HEK293 cells and were tested for IFN $\beta$ promoter activity by the Luciferase assay. Coexpression of UL48N with TRAF6, TRAF3, IRAK1, IRF7 or STING, inhibited IFN $\beta$ promoter activity whereas UL48N $\triangle$ DUB completely rescued the inhibition in case of TRAF3 and IRAK1, and partially rescued in case of TRAF6, IRF7 and STING (Figure 6b). We concluded that UL48N is able to deubiquitinate multiple signaling molecules to inhibit I-IFN production. Finally, the K63-deubiquitination activity of UL48N was tested by immunoblot analysis using various constructs such as myc-tagged TRAF6, TRAF3, IRAK1, IRF7 or STING and FLAG-tagged UL48N and UL48N $\triangle$ DUB as indicated in Figure $6 \mathrm{c}$. The result showed that UL48N hugely deubiquitinates TRAF6, TRAF3 and STING (Figure 6d) and slightly deubiquitinates IRAK1 (Supplementary Figure S3a) and IRF7 (Supplementary Figure S3b). Collectively, these results suggest that HCMV-DUB targets various key molecules of the signaling cascade to suppress I-IFN and to induce oncogenesis.

\section{Discussion}

HCMV association with various cancer tissues is wellestablished, however role of HCMV components in oncogenesis is poorly understood. Studies showed indirect roles of HCMV immediate-early proteins (IE1 and IE2) in oncogenesis, ${ }^{47-49}$ however, lacked the information on underlying molecular mechanism in oncogenesis. Our study suggests that as a consequence of overcoming anti-viral innate immune response, HCMV infection and enzymatic activity of the HCMV-DUB, in particular, may initiate early steps in oncogenesis (Figure 7). This conclusion is based on the findings stemming from HCMV-infected cells and extended to transfection experiments done with the HCMV-DUB and specific cellular anti-viral response molecules. We showed 
a qPCR
(3 dpi/dpt and 6 dpi/dpt)

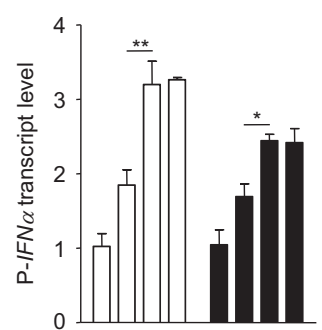

H-WT -+--++-

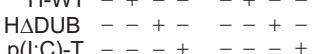

b $\quad \mathrm{qPCR} \quad$ C ( $3 \mathrm{dpi} / \mathrm{dpt}$ and $6 \mathrm{dpi} / \mathrm{dpt}$ )

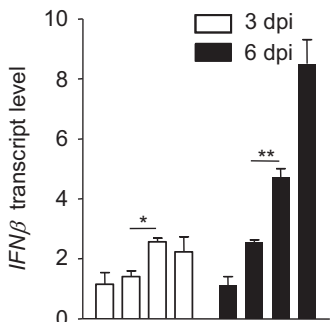

$\mathrm{H}-\mathrm{WT}$
$\mathrm{H} \triangle \mathrm{DUB}$

$H \triangle D U B$
$p(l \cdot C)-T$

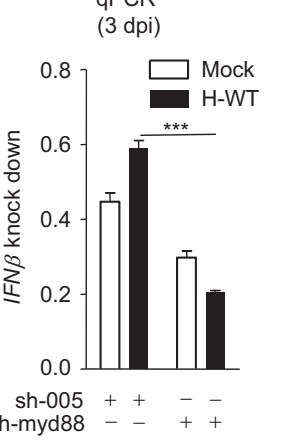

d $\quad$ qPCR

(3 dpi)

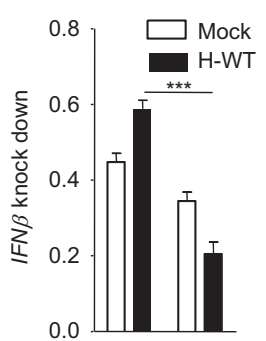

sh-005 + + - -

sh-sting - g
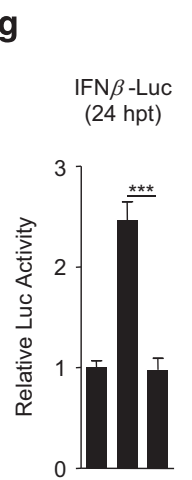

STING -

Vec +

UL48N -

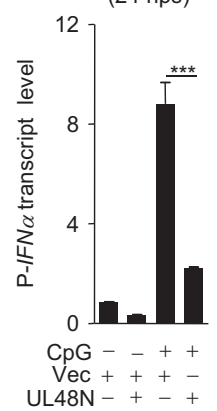

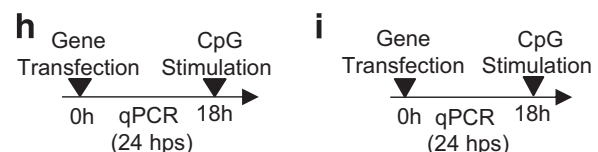

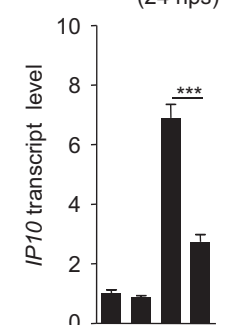

$\mathrm{CpG}--++$
$\mathrm{Vec}+++-$

UL48N - + - +

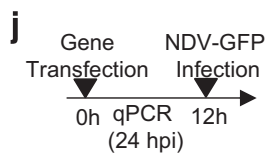

(24 hpi)

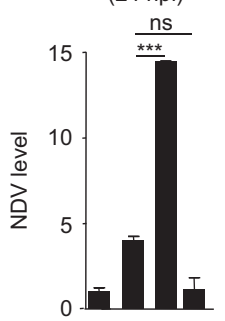

$10 \mathrm{MOINDV}-+++$

UL48N

N $\triangle D U B$

Anti-FLAG -128 KD e IFN $\alpha 4$-Luc $\mathbf{f}$ IFN $\beta$-Luc (24 hpt)

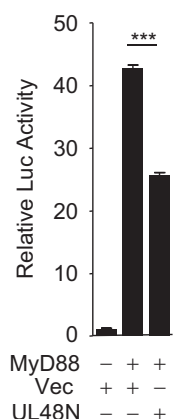

(24 hpt)

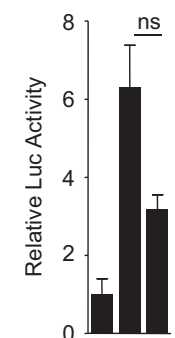

MyD88 - + +

Vec ++-

UL48N - - +
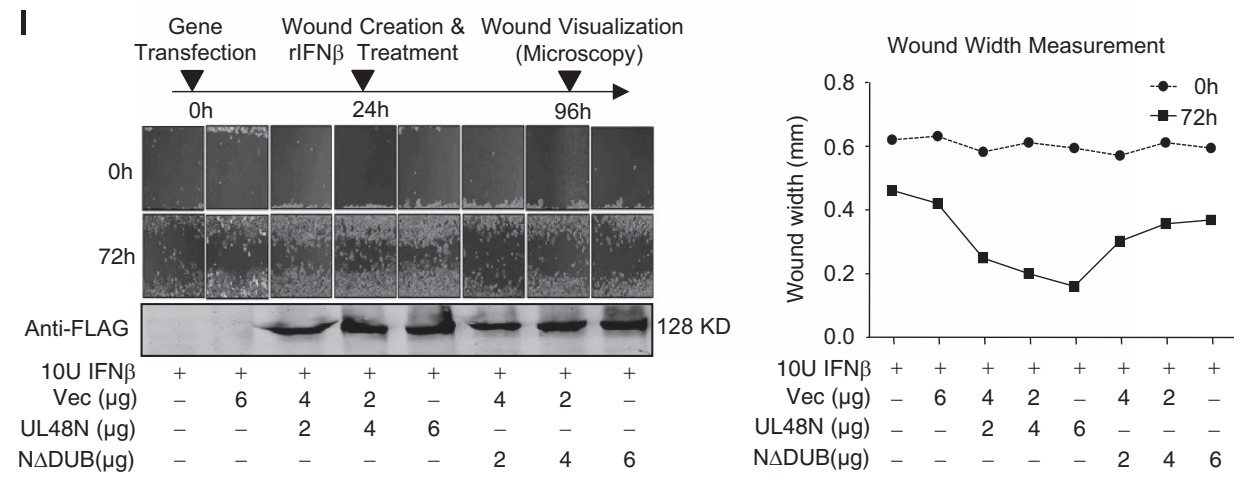

k
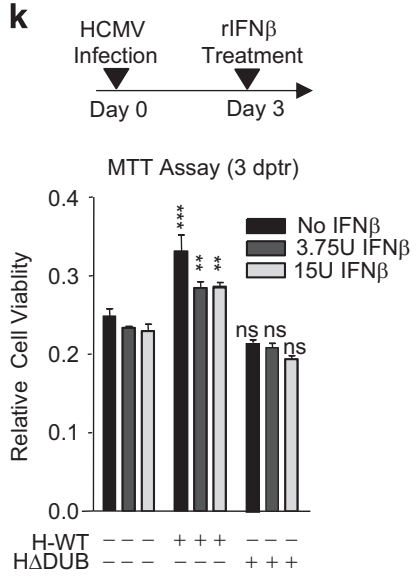

m

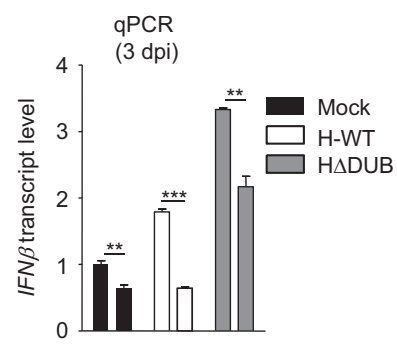

Anti-IFNAR2 n

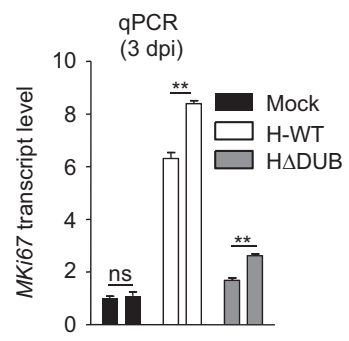

Anti-IFNAR

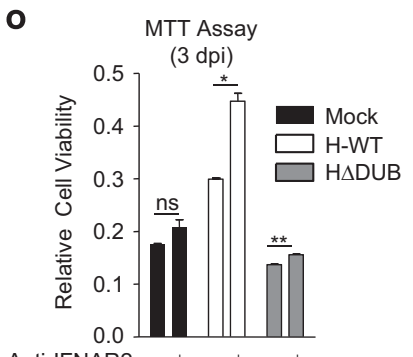

Anti-IFNAR2 
Figure 4 HCMV deubiquitinase inhibits anti-viral innate immunity to induce oncogenesis (a) Transcript of pan-interferon- $\alpha$ (P-IFN $\alpha$ ) and (b) IFN $\beta$ was measured by qPCR upon WT-HCMV (H-WT) and $\triangle$ DUB-HCMV (H $\Delta \mathrm{DUB})$ infection or poly $(\mathrm{l}: \mathrm{C})$ transfection to HFF cells, on 3 and on $6 \mathrm{dpi}$ or dpt, as indicated. (c, d) Transcript of IFN $\beta$ was measured by qPCR upon H-WT infection to myd88 (c) or sting (d) knocked down HFFs. (e-g) Luciferase assay was done for IFN $\alpha 4$ and IFN $\beta$ promoters in HEK293 cells, as depicted. (h, i) Transcript level of P-IFN $\alpha$ and IP10 was measured in HeLa cells transfected with UL48N and stimulated with CpG. (j, top) NDV transcript level was measured in IMR32 cells transiently transfected with Vec, UL48N and UL48N $\triangle D$ DUB expression plasmids as depicted. (j, bottom) Overexpression of UL48N and UL48N $\Delta$ DUB was detected by using anti-FLAG antibody. (k) MTT assay was done with HCMV-infected and rIFN- $\beta$ treated HFFs as depicted and significance of WT- or H $\Delta$ DUB infection group was calculated compared with mock. (I, left-top) Wound-healing was observed in IMR32 cells transiently transfected with increasing amount of Vec, UL48N and UL48N $\Delta$ DUB expression plasmids and treated with constant amount of rIFN- $\beta(10 \mathrm{U})$, as depicted, (I, right) wound size was measured and (I, left bottom) expression of UL48N and UL48N $\Delta \mathrm{DUB}$ was analyzed using anti-FLAG antibody. (m, $\mathbf{n}$ ) Transcript of IFN $\beta$ and MKi67 was measured by qPCR upon anti-IFNAR2 antibody treatment and WT-HCMV (H-WT) or $\triangle$ DUB-HCMV (H $\triangle \mathrm{DUB}$ ) infection to HFF cells, on 3 dpi as indicated. (o) MTT assay was done with HFF cells upon anti-IFNAR2 antibody treatment (for $4 \mathrm{~h}$ ) and WT-HCMV (H-WT) or $\triangle \mathrm{DUB}$ HCMV (H $\Delta$ DUB) infection to HFF cells, on 3 dpi as indicated. Vec: Empty Vector, N $\Delta$ DUB: UL48N $\Delta D U B, p(I: C)-T$ : Polyinosinic:polycytidylic-Transfection, dpi: days post infection, dpt: days post transfection, hpt: hours post transfection, hps: hours post-stimulation, hpi: hours post infection, dptr: days post treatment, sh: short-hairpin, rlFN: recombinant interferon, IFNAR: Type I interferon $\alpha / \beta$ Receptor. Shown results are the representative of two (a-d, and $\mathbf{h}-\mathbf{0})$ or three (e-g) independent experiments. Differences were considered statistically significant with a ${ }^{*} P$-value $<0.05$, ${ }^{* \star} P$-value $<0.01$ and ${ }^{* *} P$-value $<0.001$, ns, non-significant difference $(P$-value $>0.05)$. See also Supplementary Figure S2a-S2e

either reduced in WT-HCMV-infected HFFs or was comparable to $\triangle$ DUB-HCMV infection. Similarly, we observed that expression level of several other pro-apoptotic genes (bad and bax, bak, noxa, puma) was either reduced or remained unchanged due to infection with both, WT- and $\triangle$ DUB-HCMV in HFFs. These observations suggest that differential expressionpattern of anti- or pro-apoptotic genes might be associated with stimuli, stimuli-duration or/and cell type. In addition, it may be possible that in other cell type various other anti- or proapoptotic genes might also be involved in the cell survival signaling upon HCMV infection. Collectively, we observed majority of anti- and pro-apoptotic genes are affected due to HCMV infection in HFFs to protect cells from apoptosis as well as to bring about oncogenesis via overall promoting cell survival and carcinogenic properties.

To understand the molecular mechanism of these changes, the UL48-DUB domain was expressed in IMR32 cell line, wherein its ability to influence other characteristics of cancer cells such as proliferation, migration, glucose uptake and tissue invasion could be studied more directly. Previous studies have also shown HCMV-infected human cells to be resistant to apoptosis as well as uptake more glucose, but with little evidences of tumorigenesis or mechanisms. ${ }^{47,49-52}$

Several studies show cross-talk between oncogenesis and innate immunity wherein I-IFNs promote apoptosis via expression of pro-apoptotic genes ${ }^{10,22}$ and inhibition of antiapoptotic genes. ${ }^{10}$ Other studies suggest that overexpression of anti-apoptotic genes like bcl2 protects cells from I-IFNmediated cell death. ${ }^{27,53}$ In our study, we show WT-HCMVinfected cells express reduced I-IFNs compared with $\triangle \mathrm{DUB}$ HCMV-infected cells, which confirms HCMV-DUB as a key inhibitor of I-IFNs sythesis, that might involve in induction of oncogenic properties in HFFs. We have also shown that UL48DUB inhibits I-IFN synthesis induced through different PRRsmediated signaling pathways wherein UL48-DUB deubiquitnates signaling mediators such as TRAF6, TRAF3, IRAK1, IRF7 and STING. Interestingly, in case of TRAF6, IRF7 and STING-mediated induction of I-IFN, N $\triangle D U B-U L 48$ showed a partial repression, which also suggests a DUB-independent role of UL48N in oncogenesis. As HCMV inhibits I-IFN to counteract host innate immunity, ${ }^{54,55}$ inhibition of I-IFN synthesis by HCMV indicates its potential to establish a procancer microenvironment. In addition, studies suggest that people having a particular SNP in TLR9 (2848GA) are more susceptible to HCMV infection and cancer development. ${ }^{29,56}$
Here, we have found evidence for hypothetical dual role for HCMV-DUB, in defeating host anti-viral responses and also promoting an oncogenic state.

Several viruses encode DUBs and most of these interfere with innate immune signaling pathways. ${ }^{57-59}$ Our findings rationalize that similar to $\mathrm{HCMV}$, other DUB-encoding viruses may perturb normal cell cycle or apoptotic pathways by inhibiting I-IFN synthesis. ${ }^{58,60}$ Hence, our study provides new insight into mechanisms of virus-induced oncogenicity related to inhibition of innate immunity. Collectively, our study suggests that HCMV could be a factor leading to development of cancer through UL48-DUB in immunocompromised or immune-declined aged individuals and HCMV-DUB could be a potential therapeutic target to manage HCMV-associated cancers.

\section{Materials and Methods}

Cell lines, plasmids, antibodies and viruses. Human Embryonic Kidney (HEK293) and HeLa cell lines were obtained from American Tissue Culture Collection (ATCC). Neuroblastoma IMR32 cell line was obtained from National Centre for Cell Science (NCCS) Pune, India and HFFs were obtained from Professor Wade Gibson's Lab, Johns Hopkins, School of Medicine. All cells were cultured in Dulbecco's Modified Eagle's Medium (DMEM) supplemented with $10 \%$ FBS and $1 \times$ Anti-Biotic Anti-Mycotic, supplied by Invitrogen (Carlsbad, CA, USA) by Thermo Fisher Scientific (Waltham, MA, USA).

N-terminal of UL48 (UL48N, 1-1162 amino acids) was cloned in FLAG-tagged mammalian expression vector pCMV3Tag1a. UL48N $\triangle D$ DUB (a double mutant of C24I and H162A) UL48N $\triangle N L S$ (a single mutant of K287S) were created by using UL48N as template and cloned in pCMV3Tag1a. IRAK1, TRAF3, TRAF6, MyD88, STING were cloned in myc-tagged mammalian expression vector pCMV3Tag2a. Plasmids containing Firefly Luciferase gene under IFN $\beta$, IFN $\alpha 4$ and IFN $\alpha 6$, ISRE promoters, and Myc-tagged IRF7 were obtained from Professor Shizuo Akira's (Osaka University, Japan). HCMV genomic DNA, pCMV3Tag1a (FLAG), pCMV3Tag2a (myc) and pRL-TK were kind gifts from Professor Yan Yuan, University of Pensylvania, Philadelphia. The p21promoter-Luciferase construct was obtained from Professor Martin Walsh's Laboratory through Addgene (Cambridge, MA, USA).

Mouse raised anti-FLAG, Rabbit raised anti-myc and Rabbit raised anti-HA antibody were purchased from Sigma Aldrich (St. Louis, MO, USA). Mouse raised anti-myc antibody was purchased from Invitrogen by Thermo Fisher Scientific. IR dye labeled anti-Rabbit and anti-Mouse IgG (secondary antibody), were purchased from LI-COR. Rabbit raised anti-p53 were purchased from Invitrogen. Rabbit raised antip21, anti-BCL2, anti-Caspase-3, anti-Survivin were purchased from Cell Signaling Technology (Danvers, MA, USA), anti-BIRC3 was purchased from Santa Cruz Biotechnology (Dallas, TX, USA).

GFP-tagged (strain AD169), wild-type (H-WT) and H162A mutant (H $\Delta$ DUB, or DUB-mutant WT-HCMV and DUB-mutant HCMV were kind gifts from Professor Wade Gibson, The Johns Hopkins University School of Medicine. AD169 is extensively passaged, fibroblast adapted laboratory strain of HCMV, which is non-pathogenic; 


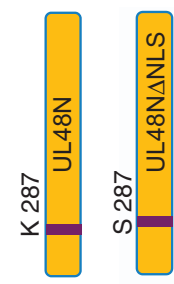

C $\mathrm{HA}-\mathrm{K} 63 \mathrm{Ub}+++$

UL48N + - -

UL48N $\triangle$ NLS - - +

IB: Anti-HA

Ubiquitination Level

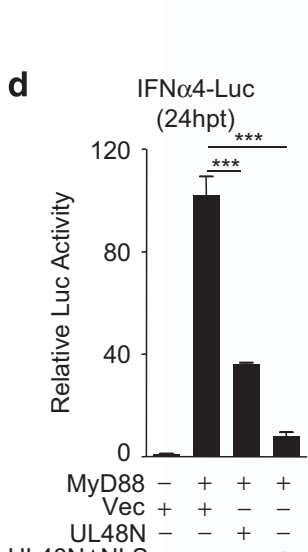

UL $48 \mathrm{~N} \triangle \mathrm{NLS}$

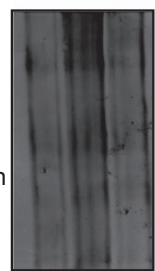

WCL e

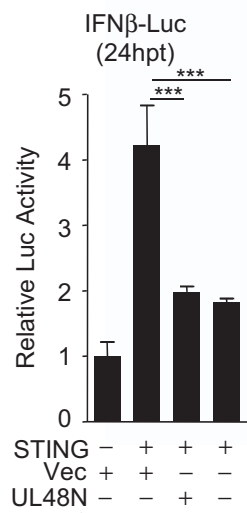

b
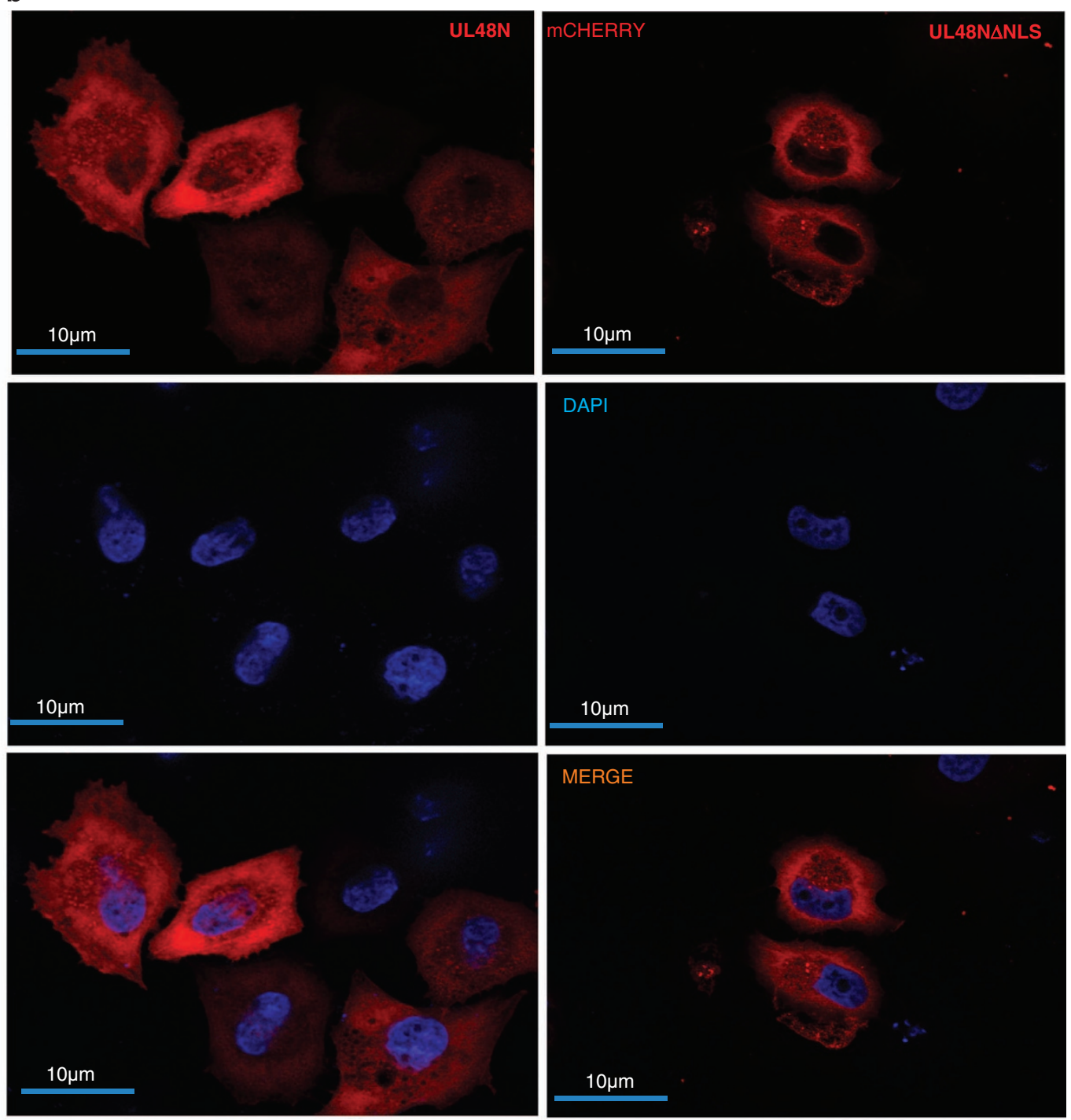

UL48N $\triangle$ NLS -

$\mathbf{f}$

$\begin{array}{ccc}\text { Gene } & \text { Wound Creation \& } & \text { Wound Visualization } \\ \text { Transfection } & \text { Microscopy } & \text { (Microscopy) }\end{array}$

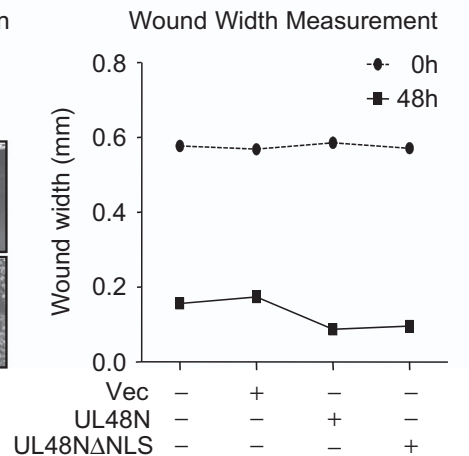

Figure 5 Nuclear Localization Sequence of UL48N is dispensable for UL48-DUB activity (a) Schematic representation of UL48N and its NLS-mutant (UL48N $\Delta$ NLS). (b) The cellular localization of mCherry-tagged UL48N and UL48N $\Delta$ NLS was observed under the fluorescence microscope at $\times 63$. Nucleus was stained with Hoechst 33342. (c) DUB activity of UL48N and UL48N $\triangle N L S$ was compared, by observing the ubiquitination status of whole cell lysates (WCL), upon overexpressing UL48N and UL48N $\triangle$ NLS in HEK293 cells, as indicated. (d, e) Luciferase assay, was done for IFN $\alpha$ or IFN $\beta$ promoter by co-transfection of MyD88 or STING and UL48N or UL48N $\Delta$ NLS expression plasmids as depicted. (f, left, right) UL48N or UL48N $\triangle$ NLS was over-expressed in IMR32 cells, wound was created and wound-healing was observed at $48 \mathrm{~h}$ and width of the wound was measured. Shown results

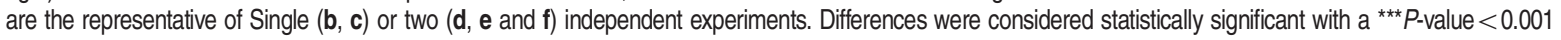


a

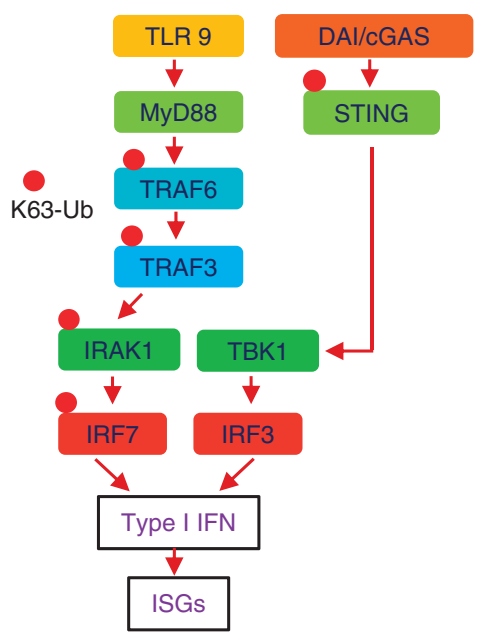

b

IFN $\beta$-Luc IFN $\quad$-Luc (24 hpt)

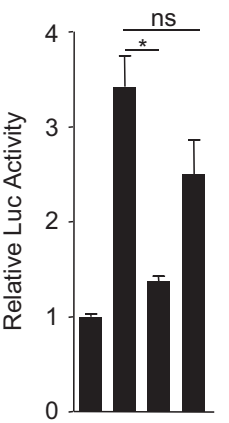

TRAF6 -+++

$\mathrm{Vec}++--$

UL48N - - + -

N $\triangle$ DUB - - - +
(24 hpt)

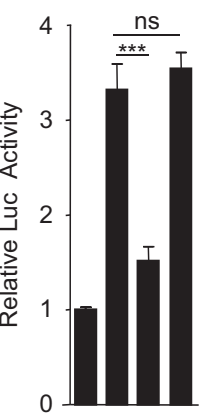

TRAF3

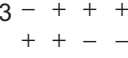

$--+$

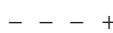

IFN $\beta$-Luc

(24 hpt)

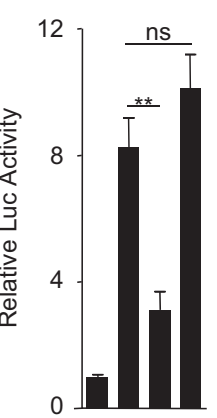

IRAK1

$$
\begin{array}{r}
1-+++ \\
++-- \\
--+- \\
---+
\end{array}
$$

IFN $\beta$-Luc (24 hpt)

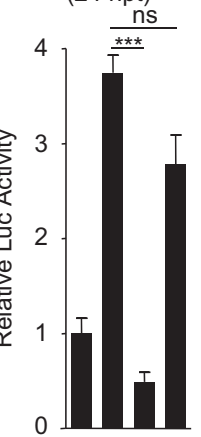

IRF7

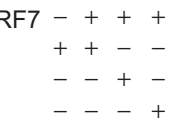

STING - + + +

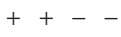

$-++$

$--+$ c

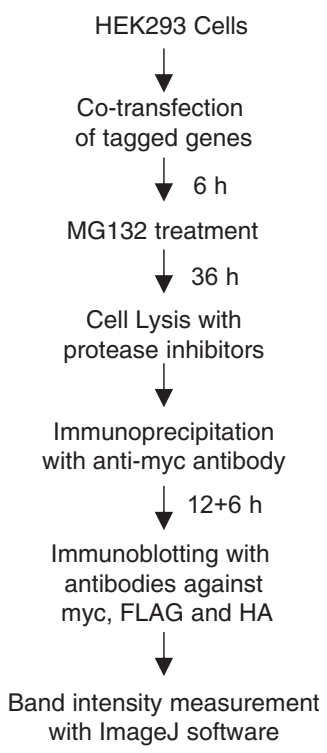

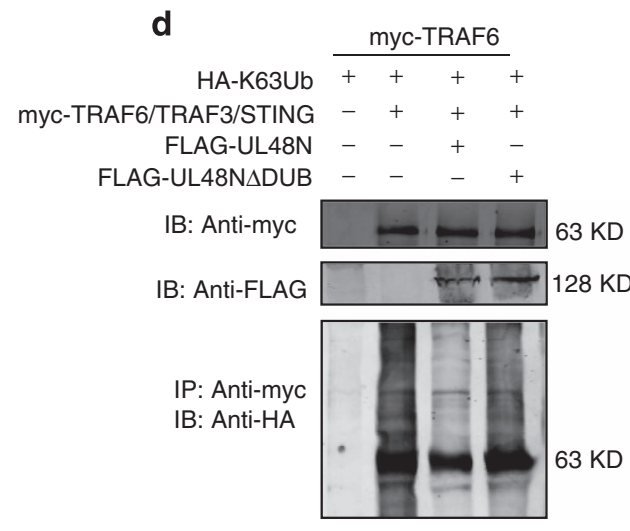

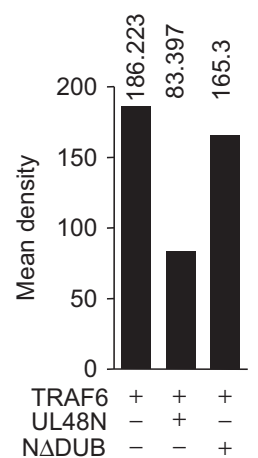

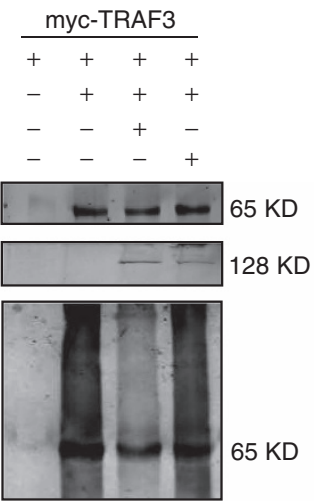
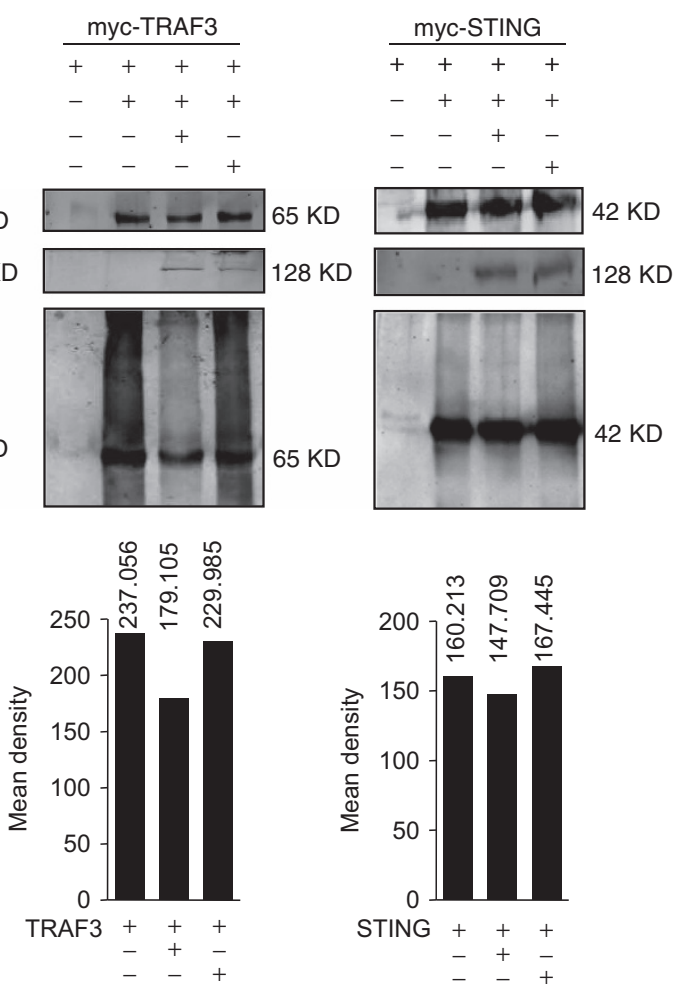

Figure 6 UL48N targets TRAF6, TRAF3 and STING for deubiqutination and inhibition of interferon synthesis. (a) Schematic representation of TLR, DNA sensing (DAI and cGAS) signaling pathways and ubiquitination of signaling molecules. (b) Luciferase assay was done for IFN $\beta$ promoter by co-transfection of TRAF6, TRAF3, IRAK1, IRF7 or STING, with UL48N or UL48N $\triangle$ DUB expression plasmids as depicted. (c, d) Ubiquitination status of TRAF6, TRAF3 and STING was analyzed in the presence of overexpression of UL48N or UL48NADUB in HEK293 cells. Co-transfection of HA-K63Ub, myc-TRAF6, myc-TRAF3 or myc-STING with FLAG-UL48N or FLAG-UL48NADUB was done as depicted. (d, top) Immunoprecipitated sample was blotted with anti-HA, anti-myc and anti-FLAG antibody to detect respective proteins, (d, bottom) Density of TRAF6, TRAF3 or STING ubiquitination was calculated and compared for each sample by using ImageJ software. Luc: Luciferase, hpt: hours post transfection, Vec: Empty Vector. Shown results are the representative of three (b) or two (d) independent experiments. Differences were considered statistically significant with a ${ }^{*} P$-value $<0.05,{ }^{* \star} P$-value $<0.01$ and ${ }^{* \star \star} P$ value $<0.001$, ns, non-significant difference $(P$-value $>0.05)$. See also Supplementary Figure S3a-S3b

NDV-GFP was a kind gift from Professor Peter Palese, Icahn School of Medicine at Mount Sinai.

Virus infection. HFFs were grown to full confluence and infected with GFPtagged HCMV at $5 \mathrm{MOI}$. At $24 \mathrm{~h}$ post infection, virus infection (\% infection) was measured through flow cytometer for FITC (GFP-HCMV). Six days post infection, CPE was observed and cells were harvested for RNA isolation. For cell cycle, Ki67 staining and apoptosis study, infection was done for three and four days respectively. IMR32 cells were infected with NDV-GFP (5 MOI) in serum free DMEM for $1 \mathrm{~h}$. Cells were then grown in reduced serum (1\% FBS) DMEM for $36 \mathrm{~h}$.

MTT assay. MTT [3-(4,5-dimethylthiazol-2-yl)-2,5- diphenyltetrazolium bromide] assay was done as described previously. ${ }^{10}$ 


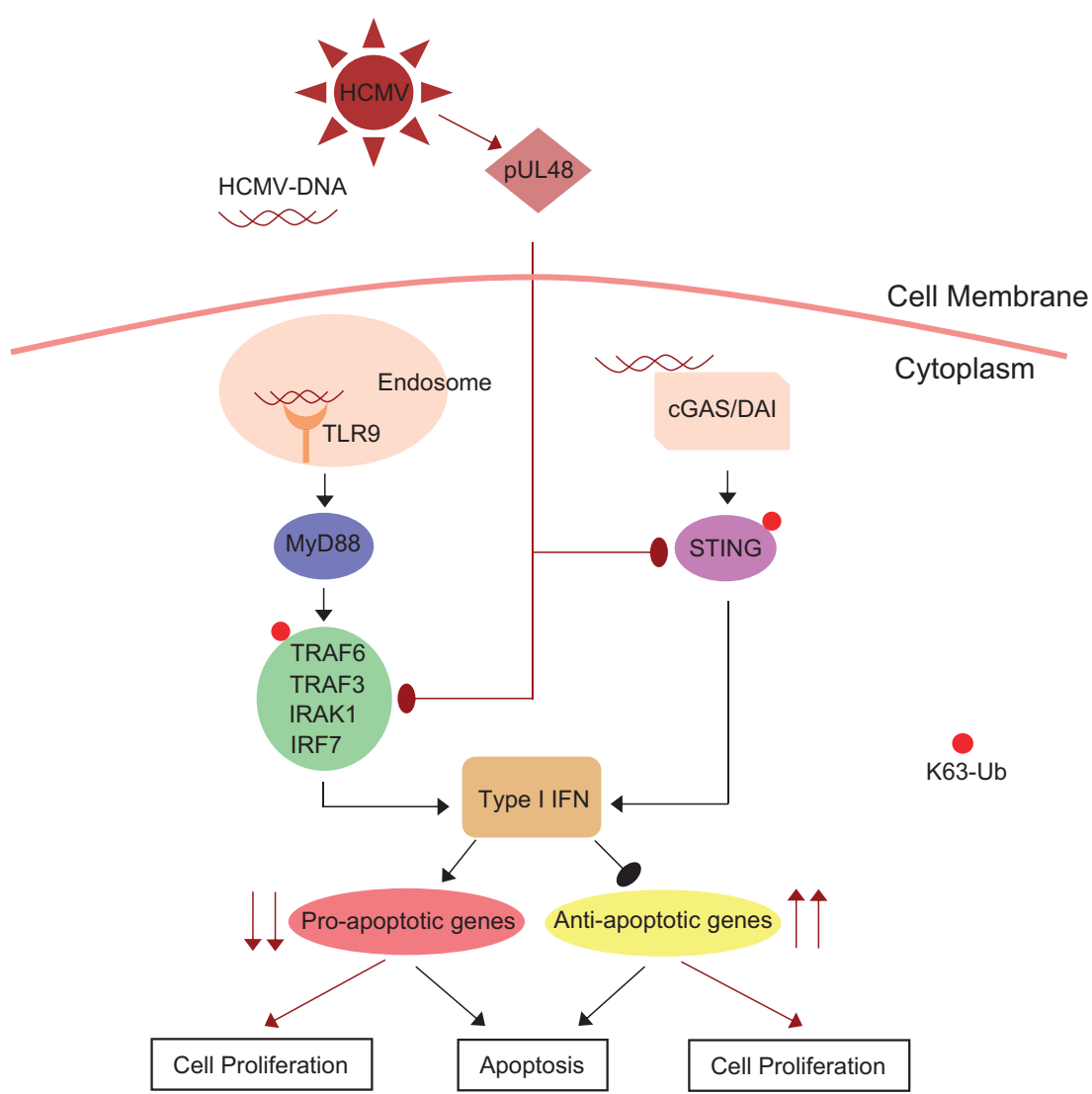

Figure 7 Induction of oncogenesis through inhibition of I-IFN synthesis by HCMV deubiqutinase (pUL48). Model showing the role of HCMV deubiquitinase in inhibiting I-IFN synthesis to promote oncogenesis. HCMV-DUB facilitates deubiquitination of TRAF6, TRAF3, IRAK1, IRF7 and STING to inhibit I-IFN synthesis, which in turn inhibits the expression of several pro-apoptotic genes and induces the expression of anti-apoptotic genes. Black arrows show normal cellular pathways. Maroon arrows show pathways during HCMV infection. Blunt arrows designate inhibitory action and pointed arrows designate stimulatory action. Red bubbles designate K63 ubiquitination of signaling molecules

Quantitative RT PCR. Designated cells were either infected with virus or transfected with respectively designated plasmids containing UL48N, UL48N $\triangle D U B$ (1 $\mu \mathrm{g} /$ well, 12 -well plate), or $1 \mu \mathrm{g} /$ well (24-well plate) of $\mathrm{p}(\mathrm{l}: \mathrm{C})$, or stimulated with $\mathrm{CpG}(0.5 \mu \mathrm{M} /$ well $)$ for indicated times. Total RNA was isolated using Trizol reagent (Ambion/Invitrogen) and CDNA was synthesized using iScript cDNA synthesis kit (BioRad, Hercules, CA, USA) as per the manufacturer's protocol. cDNA was used to analyze the transcript level in samples as indicated. Real time quantification was done using StepOne Plus Real time PCR Systems by Applied BioSystems (Foster City, CA, USA). Primers used for quantitative RT PCR are previously mentioned ${ }^{10}$ and also listed in Supplementary Table1.

Cell cycle flowcytometry. HFFs were grown to $100 \%$ confluence and infected with wild-type and DUB-mutant HCMV. After 3 days of infection cells were harvested and fixed with $70 \%$ chilled ethanol. RNaseA ( $5 \mu \mathrm{l}$ of $10 \mathrm{mg} / \mathrm{ml}$ stock) was added to ethanol and incubated at $4{ }^{\circ} \mathrm{C}$ for 30 mins. Cells were washed with $1 \mathrm{x}$ PBS and propidium iodide (PI, $10 \mu \mathrm{l}$ of $100 \mathrm{mg} / \mathrm{ml} \mathrm{stock}$ ) was added to cells and incubated Overnight at $4{ }^{\circ} \mathrm{C}$. PI-based cell cycle flow cytometric analysis was done using FACS Aria III (Becton Dickinson, Franklin Lakes, NJ, USA) and data were analyzed by using FlowJo software version 10 (FlowJo, Ashland, OR, USA).

Annexin-PE flowcytometry. HFFs were grown to $100 \%$ confluence and infected with wild-type and DUB-mutant HCMV. On second dpi, cells were treated with etoposide $(30 \mu \mathrm{M})$ and incubated for $24 \mathrm{~h}$. On third dpi cell were trypsinised, harvested, washed with $1 \times$ PBS and processed for Annexin-PE Flow Cytometery using the BD Biosciences (Haryana, India) Annexin V apoptosis kit (Cat No\# 556422) as per the manufacturer's protocol. Flowcytometric analysis was done using FACS Aria III (Becton Dickinson) and data were analyzed by using FlowJo software version 10 (FlowJo).
MKi67 staining. HCMV-infected HFFs were harvested on 3 dpi, washed with $1 \times$ PBS, fixed with $70 \%$ chilled ethanol at $-20{ }^{\circ} \mathrm{C}$ for $2 \mathrm{~h}$. Cells were washed with staining buffer (1\%FBS and $0.09 \%$ NaN3 made in $1 \times$ PBS). 1:200 dilution of APC labeled Ki67 antibody (Novus Biologicals, Littleton, CO, USA, Cat No.\# NB11089717APC) was used for staining the fixed cells at room temperature. Stained cells were analyzed for MKi67 nuclear staining using FACS Aria III (Becton Dickinson) and data were analyzed by using FlowJo software version 10 (FlowJo).

MKi67 stable cell generation and HFF knockdown. UL48N and UL48N $\triangle$ DUB constructs were prepared by subcloning into pMSCV-puro vector. Transduction of IMR32 cells was performed as described earlier (Tailor et al. 2007). In brief, viral supernatants were prepared in BOSC23 packaging cell line using construct for VSV-G. Cells were spinoculated $\left(2400 \mathrm{rpm}, 33^{\circ} \mathrm{C}, 1 \mathrm{~h}\right)$ with viral supernatants and selected using $0.2 \mu \mathrm{g} / \mathrm{ml}$ of Puromycin for $48 \mathrm{~h}$.

Knockdown experiment: HFFs were transfected with shRNA of MyD88 (Clone D1) or STING (Clone A8), obtained from Sigma Aldrich and selected on puromycin. The transcript level of myd88 and sting was analyzed through qPCR. HCMV infection (5 $\mathrm{MOI}$ ) was done to knockdown cells for 3 days and on 3 dpi cells were harvested to isolate RNA for real time analysis of IFN $\beta$ transcripts. The shRNA against GFP (sh005) was taken as knockdown control.

Transfection. Transfection was done using Lipofectamine 2000 or 3000 (Invitrogen) as per the manufacturer's protocol. For transfection related to immunoprecipitation, after $6-7 \mathrm{~h}$ post transfection, MG132 was added to the transfected cells to a final concentration of $0.5 \mu \mathrm{M}$ and incubated for further $30-$ $40 \mathrm{~h}$.

Glucose uptake assay. Glucose uptake assay was done as previously described. ${ }^{61}$ Cells were seeded at a density of $0.1 \times 10^{6}$ cells/well into 48-well plates in $200 \mu \mathrm{l}$ culture medium and incubated for $24 \mathrm{~h}$. Cells were then incubated in 
serum and glucose free DMEM for $2 \mathrm{~h}$. Serum and Glucose starved cells were treated with $10 \mu \mathrm{M}$ 2-NBDG (Invitrogen) for $10 \mathrm{~min}$ at $37^{\circ} \mathrm{C}$ and then analyzed on BD FACS Cantoll flow cytometer. 2-NBDG fluorescence was detected on FITC channel. 2-NBDG: (2-(N-(7-Nitrobenz-2 oxa-1,3-diazol-4-yl)Amino)-2-Deoxyglucose.

Luciferase based promoter Assay. HEK293 cells were transfected with empty vector, expression plasmids containing genes for either, MyD88, STING, TRAF6, TRAF3, IRAK1 or IRF7 ( $250 \mathrm{ng} / \mathrm{well}$, 24-well plate), UL48N ( $250 \mathrm{ng} / \mathrm{well}$, 24-well plate), UL48N $\triangle D U B$ or UL48N $\Delta$ NLS ( $250 \mathrm{ng} /$ well, 24 -well plate), along with $100 \mathrm{ng}$ of IFN $\alpha 4$, IFN $\alpha 6$, IFN $\beta$ and ISRE promoter containing Firefly luciferase expression plasmid and Renilla Luciferase containing plasmids (pRL-TK, $5 \mathrm{ng} /$ well) as depicted. Luciferase assay was done with total cell lysate containing abovementioned promoters and gene constructs by using Dual-Glo, Luciferase assay system (Promega, Madison, WI, USA) as per the manufacturer's protocol. Luminescence of each sample was measured by the Glomax (Promega).

Wound-healing assay. IMR32 Cells were grown at $100 \%$ confluence and transfected with designated plasmids (1 $\mu \mathrm{g} / \mathrm{well}, 12$-well plate, or as indicated). A sharp wound was created using $200 \mu$ pipette tips, $6 \mathrm{~h}$ post transfection. Wounds were visualized at $\times 10$ Sigma Aldrich objective lens under the bright field microscope and three random images were captured for few days as indicated. Wound width was measured and plotted as a line graph using GraphPad Prism Version 5.

Matrigel invasion assay. Matrigel invasion assay was done as demonstrated at http://www.abnova.com

Immunoprecipitation (IP) and Immunoblotting. For deubiquitination studies, each plasmid was taken $2 \mu \mathrm{g}$ in concentration for transfection into cells grown in $35 \mathrm{~mm}$ culture dish. Cells were treated with $0.5 \mu \mathrm{M}$ MG132, $6 \mathrm{~h}$ post transfection. Cells were harvested after $36 \mathrm{~h}$. of transfection with standard cell lysis buffer supplemented with $1 \times$ protease inhibitor cocktail (obtained from Sigma Aldrich) and $10 \mathrm{mM}$ NEM ( $N$-Ethyl Maleimide). Immunoprecipitation and Immunoblotting were done as described previously. ${ }^{62}$ Immunoblotted nitrocellulose membrane was imaged with LI-COR system.

For endogenous protein detection, mock or HCMV-infected HFFs (grown in sixwell plate) were lysed in $30 \mu \mathrm{l}$ of standard cell cell lysis buffer and $\sim 8 \mu \mathrm{g}$ of protein was loaded to each well.

Image J image analysis. Western blot densitometry: an area of interest was selected on the smeary part above the protein bands shown in the immunoblots. Mean density of the selected area was then calculated.

Microscopy. The mCherry-tagged UL48N and UL48NANLS were transfected in the HeLa cells. After $24 \mathrm{~h}$ of transfection, cells were fixed and stained with DNA staining reagent Hoechst 33342. Localization of pUL48 and pUL48N $\Delta$ NLS was visualized at $\times 63$ with Apotome-AXIO fluorescence microscope by Zeiss. HCMV infection (GFP fluorescence) was visualized with Inverted microscope Vert.A1 (AXIO) by Zeiss.

I-IFN receptor blocking. The IFNAR $\alpha / \beta$ receptor was blocked as described previously, ${ }^{63}$ using anti-IFNAR chain2 antibody (Clone\#MMHAR2, Cat\# MAB1155) from Merck. Upon blocking for $4 \mathrm{~h}$, HFFs were infected with HCMV.

Statistical analysis. Statsitical analysis was done with the help of GraphPad Prism, version 5. In the bar graph, differences between two groups were compared using an unpaired two-tailed Student's $t$-test, whereas the differences between three or more groups was calculated by using one-way analysis of variance by Newman-Keuls test. Differences were considered statistically significant with a ${ }^{*} P$-value $<0.05,{ }^{* *} P$-value $<0.01$ and ${ }^{* * *} P$-value $<0.001, \quad n s$, non-significant difference $(P$-value $>0.05)$.

\section{Conflict of Interest}

The authors declare no conflict of interest.

Acknowledgements. We thank Professor Wade Gibson for providing GFPHCMV and HFFs, and also for critically reading the manuscript; Professor Shao-Cong
Sun for providing HA-TRAF3 (Addgene), which was used as template for cloning TRAF3; Professor Shizuo Akira for all the luciferase constructs; Professor Yan Yuan for providing HCMV genomic DNA, pCMV3Tag1a, pCMV3Tag2a and pRL-TK; Professor Peter Palese for NDV-GFP and bei RESOURCES for human rIFN- $\beta$. We thank IISER Bhopal, for providing Central instrumentation facility. This study was also partly supported by the Grant for Joint Research Program of the Institute for Genetic Medicine, Hokkaido University, Japan (HK and AT). We thank DBT- State Biotech Hub, Mizoram University for infrastructural facility. PK is supported by Council of Scientific and Industrial Research (CSIR) research fellowship.

\section{Author contributions}

PK and HK conceptualized the study and designed the experiments; PK, IS and AN performed the experiments; PK, HK, IS and PT designed glucose uptake and proliferation assay; IS generated stable IMR32 cells, performed glucose uptake and proliferation assay; IS and PT helped in analyzing FACS data; AN generated DUB and NLS-mutant UL48N clones, performed few immunoblotting and few qPCR experiments; NSK helped with MKi67 FACS experiment; AT designed few experiments, analyzed data and critical read the manuscript; SN provided critical reagents for this study; PK and HK analyzed the data, and wrote the manuscript; and HK supervised the entire project.

\section{Publisher's Note:}

Springer Nature remains neutral with regard to jurisdictional claims in published maps and institutional affiliations.

1. McLaughlin-Drubin ME, Munger K. Viruses associated with human cancer. Biochim Biophys Acta 2008; 1782: 127-150.

2. Morales-Sanchez A, Fuentes-Panana EM. Human viruses and cancer. Viruses 2014; 6 : 4047-4079.

3. Landi D, Hegde M, Ahmed N. Human cytomegalovirus antigens in malignant gliomas as targets for adoptive cellular therapy. Front Oncol 2014; 4: 338.

4. Taher C, de Boniface J, Mohammad AA, Religa P, Hartman J, Yaiw KC et al. High prevalence of human cytomegalovirus proteins and nucleic acids in primary breast cancer and metastatic sentinel lymph nodes. PIOS ONE 2013; 8: e56795.

5. Herbein G, Kumar A. The oncogenic potential of human cytomegalovirus and breast cancer. Front Oncol 2014; 4: 230

6. Michaelis M, Doerr HW, Cinatl J. The story of human cytomegalovirus and cancer: increasing evidence and open questions. Neoplasia 2009; 11: 1-9.

7. Streblow DN, Dumortier J, Moses AV, Orloff SL, Nelson JA. Mechanisms of cytomegalovirus-accelerated vascular disease: induction of paracrine factors that promote angiogenesis and wound healing. Curr Top Microbiol Immunol 2008; 325: 397-415.

8. Ogasawara S, Yano H, Momosaki S, Akiba J, Nishida N, Kojiro S et al. Growth inhibitory effects of IFN-beta on human liver cancer cells in vitro and in vivo. J Interferon Cytokine Res 2007: 27: 507-516.

9. Yano H, lemura A, Haramaki M, Ogasawara S, Takayama A, Akiba J et al. Interferon alfa receptor expression and growth inhibition by interferon alfa in human liver cancer cell lines. Hepatology 1999; 29: 1708-1717.

10. Kumar S, Ingle H, Mishra S, Mahla RS, Kumar A, Kawai T et al. IPS-1 differentially induces TRAIL, BCL2, BIRC3 and PRKCE in type I interferons-dependent and -independent anticancer activity. Cell Death Dis 2015; 6: e1758.

11. Zitvogel L, Galluzzi L, Kepp O, Smyth MJ, Kroemer G. Type I interferons in anticancer immunity. Nat Rev Immunol 2015; 15: 405-414.

12. D'Arcy $P$, Wang $X$, Linder $S$. Deubiquitinase inhibition as a cancer therapeutic strategy. Pharmacol Ther 2015; 147: 32-54.

13. Zinngrebe J, Montinaro A, Peltzer N, Walczak H. Ubiquitin in the immune system. EMBO Rep 2014; 15: 28-45.

14. Yuan J, Kroemer G. Alternative cell death mechanisms in development and beyond. Genes Dev 2010; 24: 2592-2602.

15. Jarosinski K, Kattenhorn L, Kaufer B, Ploegh $\mathrm{H}$, Osterrieder N. A herpesvirus ubiquitinspecific protease is critical for efficient $T$ cell lymphoma formation. Proc Natl Acad Sci USA 2007; 104: 20025-20030.

16. Singh N, Singh AB. Deubiquitinases and cancer: a snapshot. Crit Rev Oncol Hematol 2016; 103: $22-26$

17. Fraile JM, Quesada V, Rodriguez D, Freije JM, Lopez-Otin C. Deubiquitinases in cancer: new functions and therapeutic options. Oncogene 2012; 31: 2373-2388.

18. Luise C, Capra M, Donzelli M, Mazzarol G, Jodice MG, Nuciforo $P$ et al. An atlas of altered expression of deubiquitinating enzymes in human cancer. PIOS ONE 2011; 6: e15891.

19. Wang J, Loveland AN, Kattenhorn LM, Ploegh HL, Gibson W. High-molecular-weight protein (pUL48) of human cytomegalovirus is a competent deubiquitinating protease: mutant viruses altered in its active-site cysteine or histidine are viable. J Virol 2006; 80: 6003-6012. 
20. Kim ET, Oh SE, Lee YO, Gibson W, Ahn JH. Cleavage specificity of the UL48 deubiquitinating protease activity of human cytomegalovirus and the growth of an active-site mutant virus in cultured cells. J Virol 2009; 83: 12046-12056.

21. Warburg O, Wind F, Negelein E. The metabolism of tumors in the body. J Gen Physiol 1927; 8: $519-530$.

22. Takaoka A, Hayakawa S, Yanai $\mathrm{H}$, Stoiber D, Negishi $\mathrm{H}$, Kikuchi $\mathrm{H}$ et al. Integration of interferon-alpha/beta signalling to p53 responses in tumour suppression and antiviral defence. Nature 2003; 424: 516-523.

23. Kumar R, Atlas I. Interferon alpha induces the expression of retinoblastoma gene product in human Burkitt lymphoma Daudi cells: role in growth regulation. Proc Natl Acad Sci USA 1992; 89: 6599-6603.

24. Arany I, Rady P, Tyring SK. Interferon treatment enhances the expression of underphosphorylated (biologically-active) retinoblastoma protein in human papilloma virus-infected cells through the inhibitory TGF beta 1/IFN beta cytokine pathway. Antiviral Res 1994; 23: 131-141.

25. Liedtke C, Groger N, Manns MP, Trautwein C. Interferon-alpha enhances TRAIL-mediated apoptosis by up-regulating caspase-8 transcription in human hepatoma cells. J Hepatol 2006; 44: 342-349

26. Lau E, Sedy J, Sander C, Shaw MA, Feng Y, Scortegagna M et al. Transcriptional repression of IFNbeta1 by ATF2 confers melanoma resistance to therapy. Oncogene 2015; 34: 5739-5748.

27. Karauzum SB, Yasar D, Dirice E, Imir N, Luleci G, Ozes ON. Lack of BCL-2 confers interferon-alpha sensitivity to B-cell lymphomas. Growth Factors 2007; 25: 94-100.

28. Varani S, Cederarv M, Feld S, Tammik C, Frascaroli G, Landini MP et al. Human cytomegalovirus differentially controls $B$ cell and $T$ cell responses through effects on plasmacytoid dendritic cells. J Immunol 2007; 179: 7767-7776.

29. Wujcicka W, Paradowska E, Studzinska M, Wilczynski J, Nowakowska D. Toll-like receptors genes polymorphisms and the occurrence of HCMV infection among pregnant women. Virol J 2017; $14: 64$

30. Paradowska E, Jabońska A, Studzińska M, Skowrońska K, Suski P, Wiśniewska-Ligier M et al. TLR9 $-1486 \mathrm{~T} / \mathrm{C}$ and $2848 \mathrm{C} / \mathrm{T}$ SNPs Are Associated with Human Cytomegalovirus Infection in Infants. PloS ONE 2016; 11: e0154100.

31. Paijo J, Döring M, Spanier J, Grabski E, Nooruzzaman M, Schmidt T et al. cGAS senses human cytomegalovirus and induces type I interferon responses in human monocytederived cells. PLoS Pathog 2016; 12: e1005546.

32. DeFilippis VR, Alvarado D, Sali T, Rothenburg S, Fruh K. Human cytomegalovirus induces the interferon response via the DNA sensor ZBP1. J Virol 2010; 84: 585-598.

33. Hoshino K, Kaisho T, Iwabe T, Takeuchi O, Akira S. Differential involvement of IFN-beta in Toll-like receptor-stimulated dendritic cell activation. Int Immunol 2002; 14: 1225-1231.

34. Sun L, Wu J, Du F, Chen X, Chen ZJ. Cyclic GMP-AMP synthase is a cytosolic DNA sensor that activates the type I interferon pathway. Science 2013; 339: 786-791.

35. Burdette DL, Vance RE. STING and the innate immune response to nucleic acids in the cytosol. Nat Immunol 2013; 14: 19-26.

36. Zhang K, Kumar R. Interferon-alpha inhibits cyclin E- and cyclin D1-dependent CDK-2 kinase activity associated with RB protein and E2F in Daudi cells. Biochem Biophys Res Commun 1994; 200: 522-528.

37. Vadiveloo PK, Vairo G, Novak U, Royston AK, Whitty G, Filonzi EL et al. Differentia regulation of cell cycle machinery by various antiproliferative agents is linked to macrophage arrest at distinct G1 checkpoints. Oncogene 1996; 13: 599-608.

38. Hamilton JA. CSF-1 and cell cycle control in macrophages. Mol Reprod Dev 1997; 46: 19-23.

39. Matsuoka M, Tani K, Asano S. Interferon-alpha-induced G1 phase arrest through upregulated expression of CDK inhibitors, p19Ink4D and p21Cip1 in mouse macrophages. Oncogene 1998; 16: 2075-2086.

40. Hung WC, Chuang LY. Sodium butyrate enhances STAT 1 expression in PLC/PRF/5 hepatoma cells and augments their responsiveness to interferon-alpha. Br J Cancer 1999; 80: 705-710.

41. Kroger A, Stirnweiss A, Pulverer JE, Klages K, Grashoff M, Reimann J et al. Tumor suppression by IFN regulatory factor-1 is mediated by transcriptional down-regulation of cyclin D1. Cancer Res 2007; 67: 2972-2981.

42. Pyo P, Louie B, Rajamahanty S, Choudhury M, Konno S. Possible immunotherapeutic potentiation with D-fraction in prostate cancer cells. J Hematol Oncol 2008; 1: 25.

43. Xie C, Xie DY, Lin BL, Zhang GL, Wang PP, Peng L et al. Interferon-beta gene-modified human bone marrow mesenchymal stem cells attenuate hepatocellular carcinoma through inhibiting AKT/FOXO3a pathway. Br J Cancer 2013; 109: 1198-1205.

44. Park MS, Kim BR, Kang S, Kim DY, Rho SB. The antihypertension drug doxazosin suppresses JAK/STATs phosphorylation and enhances the effects of IFN-alpha/gammainduced apoptosis. Genes Cancer 2014; 5: 470-479.
45. Chen S, Liang L, Wang Y, Diao J, Zhao C, Chen G et al. Synergistic immunotherapeutic effects of Lycium barbarum polysaccharide and interferon-alpha2b on the murine Renca renal cell carcinoma cell line in vitro and in vivo. Mol Med Rep 2015; 12: 6727-6737.

46. Brock I, Kruger M, Mertens T, von Einem J. Nuclear targeting of human cytomegalovirus large tegument protein pUL48 is essential for viral growth. J Virol 2013; 87: 6005-6019.

47. Costa H, Xu X, Overbeek G, Vasaikar S, Patro CP, Kostopoulou ON et al. Human cytomegalovirus may promote tumour progression by upregulating arginase-2. Oncotarget 2016; 7: 47221-47231.

48. Khan Z, Yaiw KC, Wilhelmi V, Lam H, Rahbar A, Stragliotto G et al. Human cytomegalovirus immediate early proteins promote degradation of connexin 43 and disrupt gap junction communication: implications for a role in gliomagenesis. Carcinogenesis 2014; 35 : $145-154$.

49. Kim J, Kwon YJ, Park ES, Sung B, Kim JH, Park CG et al. Human cytomegalovirus (HCMV) IE1 plays role in resistance to apoptosis with etoposide in cancer cell line by Cdk2 accumulation. Microbiol Immunol 2003; 47: 959-967.

50. Yu Y, Maguire TG, Alwine JC. ChREBP, a glucose-responsive transcriptional factor, enhances glucose metabolism to support biosynthesis in human cytomegalovirusinfected cells. Proc Natl Acad Sci USA 2014; 111: 1951-1956.

51. Yu Y, Maguire TG, Alwine JC. Human cytomegalovirus activates glucose transporter 4 expression to increase glucose uptake during infection. J Virol 2011; 85: 1573-1580.

52. Lukac DM, Alwine JC. Effects of human cytomegalovirus major immediate-early proteins in controlling the cell cycle and inhibiting apoptosis: studies with ts13 cells. J Virol 1999; 73 : 2825-2831.

53. Panaretakis T, Pokrovskaja K, Shoshan MC, Grander D. Interferon-alpha-induced apoptosis in U266 cells is associated with activation of the proapoptotic Bcl-2 family members Bak and Bax. Oncogene 2003; 22: 4543-4556.

54. Kumari $P$, Narayanan $S$, Kumar $H$. Herpesviruses: interfering innate immunity by targeting viral sensing and interferon pathways. Rev Med Virol 2015; 25: 187-201.

55. Browne EP, Shenk T. Human cytomegalovirus UL83-coded pp65 virion protein inhibits antiviral gene expression in infected cells. Proc Natl Acad Sci USA 2003; 100: 11439-11444.

56. Lai ZZ, Ni Z, Pan XL, Song L. Toll-like receptor 9 (TLR9) gene polymorphisms associated with increased susceptibility of human papillomavirus-16 infection in patients with cervical cancer. J Int Med Res 2013; 41: 1027-1036.

57. Clementz MA, Chen Z, Banach BS, Wang Y, Sun L, Ratia K et al. Deubiquitinating and interferon antagonism activities of coronavirus papain-like proteases. J Virol 2010; 84: 4619-4629.

58. Inn KS, Lee SH, Rathbun JY, Wong LY, Toth Z, Machida K et al. Inhibition of RIG-I-mediated signaling by Kaposi's sarcoma-associated herpesvirus-encoded deubiquitinase ORF64. J Virol 2011; 85: 10899-10904.

59. Kumari P, Kumar H. Viral deubiquitinases: role in evasion of anti-viral innate immunity. Crit Rev Microbiol 2017 (doi:10.1080/1040841X.2017.1368999).

60. Saito S, Murata T, Kanda T, Isomura H, Narita $Y$, Sugimoto A et al. Epstein-Barr virus deubiquitinase downregulates TRAF6-mediated NF-kappaB signaling during productive replication. J Virol 2013; 87: 4060-4070.

61. Zou C, Wang Y, Shen Z. 2-NBDG as a fluorescent indicator for direct glucose uptake measurement. J Biochem Biophys Methods 2005; 64: 207-215.

62. Sathish N, Zhu FX, Yuan Y. Kaposi's sarcoma-associated herpesvirus ORF45 interacts with kinesin-2 transporting viral capsid-tegument complexes along microtubules. PLOS Pathog 2009; 5: e1000332.

63. Choi HJ, Lui A, Ogony J, Jan R, Sims PJ, Lewis-Wambi J. Targeting interferon response genes sensitizes aromatase inhibitor resistant breast cancer cells to estrogen-induced cell death. Breast Cancer Res 2015; 17: 6.

Cell Death and Disease is an open-access journal published by Nature Publishing Group. This work is licensed under a Creative Commons Attribution 4.0 International License. The images or other third party material in this article are included in the article's Creative Commons license, unless indicated otherwise in the credit line; if the material is not included under the Creative Commons license, users will need to obtain permission from the license holder to reproduce the material. To view a copy of this license, visit http://creativecommons.org/licenses/by/4.0/

(C) The Author(s) 2017 